\title{
Mutation, Witten index, and quiver invariant
}

\author{
Heeyeon Kim, ${ }^{a}$ Seung-Joo Lee ${ }^{b}$ and Piljin $\mathrm{Yi}^{c}$ \\ ${ }^{a}$ Perimeter Institute for Theoretical Physics, \\ 31 Caroline Street North, Waterloo, N2L 2Y5, Ontario, Canada \\ ${ }^{b}$ Department of Physics, Robeson Hall, Virginia Tech, \\ Blacksburg, VA 24061, U.S.A. \\ ${ }^{c}$ School of Physics, Korea Institute for Advanced Study, \\ Seoul 130-722, Korea \\ E-mail: heeyeon.kim@perimeterinstitute.ca, seungsm@vt.edu, \\ piljin@kias.re.kr
}

Abstract: We explore Seiberg-like dualities, or mutations, for $\mathcal{N}=4$ quiver quantum mechanics in the context of wall-crossing. In contrast to higher dimensions, the 1d Seibergduality must be performed with much care. With fixed Fayet-Iliopoulos constants, at most two nodes can be mutated, one left and the other right, mapping a chamber of a quiver into a chamber of a mutated quiver. We delineate this complex pattern for triangle quivers and show how the Witten indices are preserved under such finely chosen mutations. On the other hand, the quiver invariants, or wall-crossing-safe part of supersymmetric spectra, mutate more straightforwardly, whereby a quiver is mapped to a quiver. The mutation rule that preserves the quiver invariant is different from the usual one, however, which we explore and confirm numerically.

KEywords: Supersymmetry and Duality, String Duality, Duality in Gauge Field Theories, Solitons Monopoles and Instantons

ArXiv EPrint: 1504.00068 


\section{Contents}

1 Introduction $\quad 1$

2 Quivers and quiver mutations $\quad 4$

$\begin{array}{lll}3 & \Omega_{\mathrm{Q}}(\zeta) \text { via Localization: summary of HKY } & 7\end{array}$

4 Wall-crossing and quiver invariants $\quad 10$

5 Mutation $\mu_{k}$ on Witten index $\Omega_{\mathrm{Q}}(\zeta) \quad 14$

$\begin{array}{lll}5.1 & \text { Mutations and chambers } & 14\end{array}$

$\begin{array}{ll}5.2 \text { A numerical check and a subtlety } & 16\end{array}$

$\begin{array}{ll}5.3 \text { Mutation invariance of Witten index } & 18\end{array}$

6 Mutation $\tilde{\mu}_{k}$ on quiver invariant $\left.\Omega_{\mathrm{Q}}\right|_{\operatorname{Inv}} \quad 24$

$\begin{array}{llr}7 & \text { Summary } & 27\end{array}$

$\begin{array}{ll}\text { A Quiver invariants from direct index computations } & 27\end{array}$

\section{Introduction}

Quiver quantum mechanics, and more generally gauged linear sigma models quantum mechanics (1d GLSM) with four or less supersymmetries, exhibit wall-crossing behavior where the Witten index jumps discontinuously under continuous deformation of Fayet-Iliopoulos constants $\zeta$. With $\mathcal{N}=4$ supersymmetry, this is directly connected to the wall-crossing of Seiberg-Witten theories [1-3] via Calabi-Yau compactification of type II string theories. Such discontinuities have been studied in many different approaches in the past. The fundamental mechanism of BPS state disappearance in $4 \mathrm{~d}$ was understood fairly early via multi-center nature of generic BPS states [4] which was followed by explicit state counting and wall-crossing of multi-center BPS bound states in the weakly coupled regime of rank two or higher gauge theories [5-7] and then later in the supergravity or Calabi-Yau setting [8-10].

These early works inspired two different approaches to the general wall-crossing problems. One resorted to more mathematical reformulation, culminating in the KontsevichSoibelman wall-crossing formulae [11, 12]. This is suitable for noncompact Calabi-Yau examples, e.g., Seiberg-Witten theories, and was later further clarified and expanded via compactified $(2,0)$ theory $[13,14]$. The other, more faithful to the physical picture of multicenter BPS states, was developed by and large parallel to the former. The latter resulted in 
a very comprehensive and universal index formulae [15-18], from which wall-crossing formulae followed. The latter, when restricted appropriately to fit the smaller scope of the former, has been shown to be solutions to the Kontsevich-Soibelman wall-crossing algebra [19].

Despite such a long history and several breakthroughs, there are some important questions remaining. For example, while we have several different wall-crossing formulae and index formulae, actual evaluation of them in examples beyond $4 \mathrm{~d}$ rank one theories are hardly understood at a systematic level. Also, beyond such more technical issues, there is also a conceptual mystery surrounding part of supersymmetric spectra that remain robust across walls of marginal stability. In the Calabi-Yau setting, this question appears to be essential to complete classification and counting of supersymmetric cycles of compact Calabi-Yau three-folds, and in particular to microstate counting of $4 \mathrm{~d} \mathcal{N}=2$ BPS black holes.

To explain these wall-crossing-safe states, let us come back to $1 \mathrm{~d}$ quiver quantum mechanics, which are low energy dynamics of D3-branes wrapped on special Lagrange cycles of Calabi-Yau three-fold $[9,20]$. Such quiver quantum mechanics has resurfaced more directly from Seiberg-Witten theories as well; one recent is via low energy dynamics of BPS solitons in strongly coupled regimes [18, 21], while another is from realization of SeibergWitten theory as $(2,0)$ theories compactified on Riemannian surfaces with punctures [13, 22, 23]. Wall-crossing of 4d BPS states then translates to appearance and disappearance of supersymmetric vacua of such $1 \mathrm{~d}$ quiver theories.

For a simpler class of quivers like two-node Kronecker quivers, all supersymmetric vacua disappear simultaneously across a single wall at $\zeta=0$. Whenever a quiver comes with a superpotential, however, a subtlety arises. Spectrum is split into part that disappear at such walls and part that remain robust everywhere in FI constant space [10, 24-26]. As we will review in a later section, the latter states are all angular momentum, or $\mathrm{SU}(2)_{R}$ singlets $[25,26]$, and serve as building blocks in the multi-center picture or the Coulombic picture, of the wall-crossing $[28,29]$. The latter class of states should exist even when all FI constants are set to zero, and have been dubbed the quiver invariant [25, 26], for an obvious reason; these states, or part of Witten index that captures them, are invariant properties of the quiver itself rather than those of individual chambers with distinct Witten indices.

Existence of such wall-crossing-safe states implies that the wall-crossing formulae are nowhere enough for counting ground states of quiver quantum mechanics, or equivalently counting BPS spectra in four dimensions. For the Kontsevich-Soibelman wall-crossing formulae, in fact, the quiver invariants should be regarded as input data rather than solution to their algebraic constraint. In $4 \mathrm{~d}$ context, the quiver invariant seems to count degeneracy of single-center $\mathcal{N}=2$ BPS black holes [26], which also tells us that for black hole microstate counting, it is not the wall-crossing pattern that matters but rather one must compute Witten indices and the quiver invariants more directly. Beyond simple cases like $\mathrm{SU}(2)$ Seiberg-Witten, therefore, the need for direct counting of Witten indices is all the more pressing.

Equivariant Witten index counting for general $\mathcal{N} \geq 2$ gauged quantum mechanics has been established in a recent work [30], where the wall-crossing in $\zeta$ space is also captured and accounted for correctly. Although actual evaluation, some $r$-dimensional contour integrals, 
is riddled with subtleties and also mired by heavy computational cost as rank $r$ grows, this result, to which we will refer as HKY, represents the most comprehensive approach to counting supersymmetric ground states of 1d GLSM. It represents the first systematic and comprehensive counting method; although there had been systematic approaches such as that of Reineke [27] or those based on Coulombic approximation [16, 18], these are effectively restricted to quivers without superpotentials and in particular cannot count black hole microstates by themselves. This new approach supersedes existing geometrical methods such as the Abelianization scheme reviewed in ref. [31], and has been used for various nontrivial examples. For low-rank or Abelian examples, the prescription is very effective and Witten indices have been computed for many $\mathcal{N}=2,4$ GLSM's.

Computation of high rank non-Abelian GLSM's, other than some of very simple quivers, remains technically challenging, however. ${ }^{1}$ On the other hand, a very suggestive scaling behavior with growing intersection numbers has been seen in the wall-crossing-safe part of spectrum [10, 21, 24]. This could be related to Witten indices of high rank quivers in two possible ways. One is via the MPS expansion which expresses the index of a high rank quiver via a partition sum of the rank vector where high rank often translates to the high intersection numbers in the computational middle steps. Another possibility is the so-called mutation map, which can preserve Witten index under favorable circumstances while mixing up rank vectors and intersection numbers.

The mutation, which is a form of Seiberg-duality for the quiver quantum mechanics, has been very successfully used for obtaining BPS spectra of rank-one Seiberg-Witten theories by Alim et al. [23] who argued how two different-looking quivers, with very different ranks, can possess chambers of the same Witten indices and explained how two such can be viewed as a mere change of basis. The basis element in question can be either a specific set of simple dyons for Seiberg-Witten theory, or a set of special Lagrangian submanifolds for Calabi-Yau three-fold. Thus, one immediate problem is to verify the proposed mutation invariance against explicit Witten index counting. Because the mutation always acts on a single node at a time and transforms the adjacent nodes by the connecting arrows, the simplest prototype where all the subtleties of mutation can be seen is the cyclic triangle quivers where each node is connected to a pair of nodes each with ingoing and outgoing arrows. One main objective of this note is to study this class of quivers in detail and demonstrate how mutation map manifests in HKY's Witten index counting.

This mutation map is, however, rather specific in that it requires certain inequalities among FI constants, $\zeta$. Because of this, the map cannot map all physical chambers of a quiver to those of one mutated quiver. Chamber by chamber, allowed mutations are generically all different. While the mutation can represent a powerful method for relating quivers of different ranks and intersection numbers, this severe $\zeta$-dependence is subtle enough to hinder most practical applications generally. On the other hand, such subtleties turn out to be absent as far as quiver invariants go. As noted above, for general quivers that accept superpotential, the notion of the quiver invariant has emerged as key ingredient

\footnotetext{
${ }^{1}$ For the simple Kronecker quivers, the large-rank scaling behavior has been also obtained with help of this approach [32-34], although the scaling behavior found here is not related to that of $\mathcal{N}=2$ BPS black holes but rather intrinsic to rank 2 or higher field theory BPS states.
} 
to understanding of the spectra $[25,26,28,29,31]$. Because the quiver invariant is a basic property of a quiver, independent of chamber choices, we can anticipate that the mutation rule preserving quiver invariant, if it exists at all, should not be mired by FI constants. However, the usual mutation rule that preserves Witten index chamber-wise is clearly inadequate for this as one immediately sees counterexamples where the mutated quiver and the original quiver have two very different chamber structures.

It turns out that the relevant mutation rule for the quiver invariant is identical to the usual mutation rule, except that it shifts the rank of the mutating node differently as

$$
N_{k} \quad \rightarrow \quad-N_{k}+\min \left(N_{f}^{(k)}, N_{a}^{(k)}\right),
$$

where $N_{f}^{(k)}$ and $N_{a}^{(k)}$ are, respectively, the total number of chiral fields in the fundamental representations and the total number of chiral fields in the anti-fundamental representations, with respect to $\mathrm{U}\left(N_{k}\right)$. This action is different from the usual mutation rule, yet preserves the quiver invariants. Because of the chamber-independent nature of the quiver invariant, this mutation on quiver invariant can act on any node of the quiver, regardless of $\zeta$ values.

In section 2, we overview the quiver data and the quiver mutations. Here we will introduce a few manifestations of mutation maps with different action on ranks but with a common action on adjacency matrix. One of them, to be distinctly denoted as $\tilde{\mu}$, will turn out to be the right action that preserves the quiver invariant. Section 3 is devoted to a brief review of HKY index formulae for gauge quantum mechanics, which is our main tool for checking how mutation acts on Witten indices and quiver invariants. After a review of wall crossing, Witten index, and quiver invariant in section 4, we move in section 5 to ordinary mutations $\mu$ and test how they preserve Witten indices selectively, using HKY's Witten index formulae. Section 6 discusses mutation on quiver invariant, given by the alternate action $\tilde{\mu}$, and makes predictions for several sequences of triangle quivers, numerical confirmations of which can be found in appendix A.

\section{Quivers and quiver mutations}

The quiver mutation rule takes a supersymmetric quiver theory with four supercharges and maps it to another such theory with different gauge group and matter content. More specifically, a quiver theory is specified by the following set of data:

- The nodes, labeled by $i$, with ranks $N_{i}$. Each node represents a vector multiplet with the gauge group $\mathrm{U}\left(N_{i}\right)$.

- The adjacency matrix, $b=\left[b_{i j}\right]$, which counts the arrows from node $i$ to node $j$. Positive $b_{i j}$ counts the chirals in the bifundamental representation, $\left(\bar{N}_{i}, N_{j}\right)$.

- Fayet-Iliopoulos (FI) constant, $\zeta_{i}$, for each node. For this note we take the normalization for $\zeta$ 's such that FI term in the Lagrangian is of the form

$$
-\zeta_{i} \int d t \operatorname{tr} D_{i}
$$

where $D_{i}$ is the auxiliary field in the gauge multiplet of $\mathrm{U}\left(N_{i}\right)$. 
- $R$-charge assignment $R_{i j}$ for chiral multiplets.

Recall that the quiver quantum mechanics would be a low energy dynamics of BPS state of total charge $\Gamma=\sum_{i} N_{i} \gamma_{i}$, of some $4 \mathrm{~d} \mathcal{N}=2$ theories [9]. The simplest setting where quiver quantum mechanics emerge is type IIB theory compactified on a CalabiYau three-fold. The effective theory in the remaining four dimensions carries $\mathcal{N}=2$ supersymmetry, and the BPS states thereof are realized as D3-branes wrapped on special Lagrange subcycles of the Calabi-Yau. When the cycle is rigid, as with $S^{3}$, the vector multiplet on the D3-brane reduces to quantum mechanical vector multiplet whose content is the same as $\mathcal{N}=1$ vector multiplet in four dimensions. ${ }^{2}$ We denote the bosonic part of the multiplet as

$$
\left(A_{0}, x_{1}, x_{2}, x_{3}\right)
$$

where the latter three transform under $\mathrm{SU}(2)_{R} R$-symmetry as triplet. Generators of this $\mathrm{SU}(2)_{R}$ are denoted as $J_{1,2,3}$. In addition there is also $\mathrm{U}(1)_{R}$ symmetry which is inherited from its four-dimensional reincarnation. We denote its half-integral generator by $I$.

When we view the quiver theory as the dynamics of D-branes wrapped on supersymmetric cycles in a Calabi-Yau three-fold, with the charge label $\gamma$ 's, the adjacency matrix, $b=\left[b_{i j}\right]$, of the quiver counts their intersections as $b_{i j}=\left\langle\gamma_{i}, \gamma_{j}\right\rangle$, whereby $b$ is manifestly an antisymmetric matrix. Finally, $\zeta_{i}$ is related to the phase of the central charge of the cycle $\gamma_{i}$.

The quiver mutation maps a quiver $\mathrm{Q}=(N ; b)_{\zeta}^{R}$ to another quiver $\widehat{\mathrm{Q}}=(\widehat{N} ; \hat{b})_{\hat{\zeta}}^{\hat{R}}$. Mathematical literatures usually start with mutation rule for the matrix $b$, but for our purpose it is more transparent to start with mutation of the underlying charges $\gamma_{i}$. For each node, say, for node $k$, one can define two different mutation maps $\mu_{k}^{L, R}$ which can be understood most easily via their action on $\gamma_{i}$ 's. For the left mutation on node $k$, we have

$$
\mu_{k}^{L}\left(\gamma_{i}\right)=\left(\begin{array}{cc}
-\gamma_{k} & i=k \\
\gamma_{i}+\left[b_{k i}\right]_{+} \gamma_{k} & \text { otherwise }
\end{array}\right.
$$

where $[b]_{+}$is $b$ for positive $b$ and zero otherwise. The right mutation is a mirror image of this,

$$
\mu_{k}^{R}\left(\gamma_{i}\right)=\left(\begin{array}{cc}
-\gamma_{k} & i=k \\
\gamma_{i}+\left[b_{i k}\right]_{+} \gamma_{k} & \text { otherwise }
\end{array}\right.
$$

Giving the mutation rule to $\gamma$ 's first has the advantage that the rule on $\zeta$ follows automatically as,

$$
\mu_{k}^{L}\left(\zeta_{i}\right)=\left(\begin{array}{cc}
-\zeta_{k} & i=k \\
\zeta_{i}+\left[b_{k i}\right]_{+} \zeta_{k} & \text { otherwise }
\end{array}\right.
$$

\footnotetext{
${ }^{2}$ When the special Lagrange cycle is not rigid, there could be further chiral multiplets, such as in the adjoint representation, although in this note we won't consider such cases.
} 

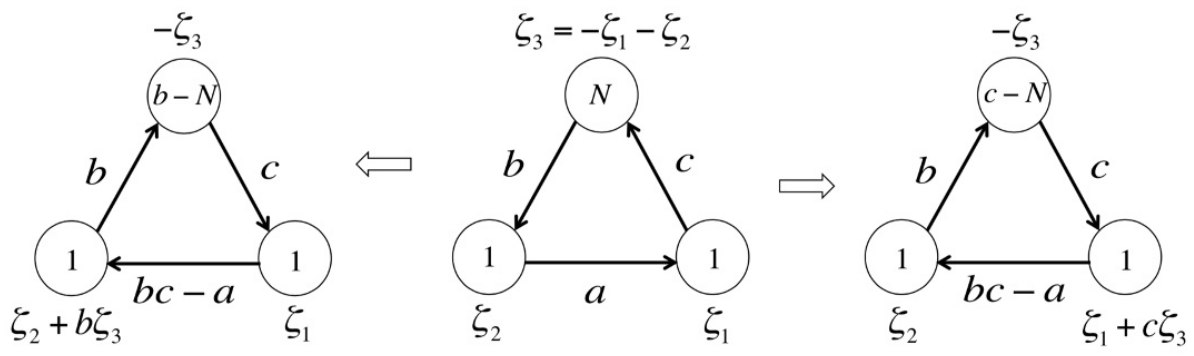

Figure 1. The left and the right mutations $\mu_{3}^{L, R}$ on node 3 for $(1,1, N)$ quivers. The integers inside circles are ranks, while the FI constants are displayed next to them.

and

$$
\mu_{k}^{R}\left(\zeta_{i}\right)=\left(\begin{array}{cc}
-\zeta_{k} & i=k \\
\zeta_{i}+\left[b_{i k}\right]_{+} \zeta_{k} & \text { otherwise }
\end{array}\right.
$$

Both of these two mutations on $\gamma_{i}$ 's lead to a common rule for $b$ as

$$
\mu_{k}\left(b_{i j}\right)=\left(\begin{array}{cl}
-b_{i j} & \text { if } i=k \text { or } j=k \\
b_{i j}+\operatorname{sgn}\left(b_{i k}\right)\left[b_{i k} b_{k j}\right]_{+} & \text {otherwise }
\end{array}\right.
$$

where we dropped the superscript since the left and the right mutations lead to a common rule. See figure 1 for an illustration. This common rule on the adjacency matrix is the usual starting point for the cluster algebra. Shift of $R$-charges, $\mu_{k}^{L, R}\left(R_{i j}\right)$, is somewhat ambiguous, due to possible mixing with gauge and flavor charges, some aspects of which will be discussed in section 5 .

When we try to apply the above mutation rule to quivers with loops, it is important to restrict to the set of quivers without 1-cycles nor 2-cycles, where the 1-cycles refer to arrows start and end at the same node, and the 2-cycles refer to two non-canceling arrows with opposite direction between two nodes. Also, the superpotential is assumed to be generic but consistent with the gauge symmetry and $R$-symmetry. The latter implies that $W$ is of charge 2 with respect to the $\mathrm{U}(1)_{R}$ in the convention where $R$-charges of supercharges are \pm 1 . One underlying assumption in the above is that we pair-annihilated chirals of mutual charge conjugate by assigning appropriate $R$-charges to them to allow for a bilinear term in $W$, which lifts them pairwise from the low energy dynamics.

Finally, the mutated quiver needs the rank data $\widehat{N}_{i}=\mu_{k}\left(N_{i}\right)$. One natural prescription is to keep $\Gamma \equiv \sum_{i} N_{i} \gamma_{i}$ invariant under the mutation, for which we have

$$
\mu_{k}^{L}\left(N_{i}\right)=\left(\begin{array}{cl}
-N_{k}+\sum_{j}\left[b_{k j}\right]_{+} N_{j} & i=k \\
N_{i} & \text { otherwise }
\end{array}\right.
$$

and

$$
\mu_{k}^{R}\left(N_{i}\right)=\left(\begin{array}{cl}
-N_{k}+\sum_{j}\left[b_{j k}\right]_{+} N_{j} & i=k \\
N_{i} & \text { otherwise }
\end{array}\right.
$$


Note that these two result is the same rule if $\sum_{j}\left[b_{j k}\right]_{+} N_{j}=\sum_{j}\left[b_{k j}\right]_{+} N_{j}$. For example, anomaly cancelation condition of $4 \mathrm{~d} \mathcal{N}=1$ theories of quiver type demands precisely this identity for each and every node, and the familiar Seiberg duality map on $N_{i}$ coincides with either of $\mu_{k}^{L, R}$.

There is another natural choice of mutation rule on $N_{i}$ 's: mutate the total charge $\Gamma=\sum_{i} N_{i} \gamma_{i}$ as if it is one of nodes in the quiver [39], i.e.,

$$
\tilde{\mu}_{k}^{L}(\Gamma)=\left(\begin{array}{cl}
-\Gamma & \Gamma=n \gamma_{k}, \quad n \in \mathbf{Z}_{+} \\
\Gamma+\left[\left\langle\gamma_{k}, \Gamma\right\rangle\right]_{+} \gamma_{k} & \text { otherwise }
\end{array}\right.
$$

and

$$
\tilde{\mu}_{k}^{R}(\Gamma)=\left(\begin{array}{cl}
-\Gamma & \Gamma=n \gamma_{k}, \quad n \in \mathbf{Z}_{+} \\
\Gamma+\left[\left\langle\Gamma, \gamma_{k}\right\rangle\right]_{+} \gamma_{k} & \text { otherwise }
\end{array}\right.
$$

where we introduced the notation $\tilde{\mu}$ to emphasize that the shifts of $N_{i}$ 's are different. Interestingly, their action, when translated to that on $N_{i}$, boils down to a common rule,

$$
\tilde{\mu}_{k}^{L, R}\left(N_{i}\right)=\left(\begin{array}{cl}
-N_{k}+\min \left(\sum_{j}\left[b_{j k}\right]_{+} N_{j}, \sum_{j}\left[b_{k j}\right]_{+} N_{j}\right) & i=k \\
N_{i} & \text { otherwise }
\end{array}\right.
$$

Otherwise $\tilde{\mu}$ 's act on $\gamma, b, \zeta$, in the same way as $\mu$ 's. We will later see that this modified mutation preserves the quiver invariant.

\section{$3 \Omega_{\mathrm{Q}}(\zeta)$ via Localization: summary of HKY}

As a preliminary, we will review the HKY index formula for the quiver quantum mechanics. The equivariant Witten index of interest is

$$
\Omega_{\mathrm{Q}}(\zeta)=\lim _{\beta \rightarrow \infty} \operatorname{tr}\left[(-1)^{2 J_{3}} \mathbf{y}^{2 J_{3}+2 I} e^{-\beta H(\zeta)}\right],
$$

where we fixed the usual sign ambiguity of the index by choosing $(-1)^{F}=(-1)^{2 J_{3}}$. When we do this we should take care to remove the center of mass part of the low energy dynamics, which is to say, to remove one overall $\mathrm{U}(1)$ decoupled from the rest of the dynamics.

For GLSM with compact classical moduli space, the localization procedure produces relatively compact finite integration over vector multiplet zero modes. Denoting collectively by $u=\beta\left(\bar{x}_{3}+i \bar{A}_{0}\right)$ the zero modes of Cartan part of the vector multiplet, the Witten index for $1 \mathrm{~d} \mathcal{N}=4$ GLSM is compactly expressed as a residue integral of the following expression $[30]^{4}$

$$
g(u)=\prod_{A} g_{\text {gauge }}^{(A)}(u) \prod_{I} g_{\text {chiral }}^{(I)}(u)
$$

\footnotetext{
${ }^{3}$ The mutation rule for the ranks can differ for different theories in various dimensions. See refs. [35-38] for $2 \mathrm{~d}$ and $3 \mathrm{~d}$ examples.

${ }^{4}$ See also refs. [40, 41] for related discussions.
} 
which comes from one-loop determinant of nonzero modes. For instance, each gauge sector, labeled by $A$, contributes

$$
g_{\text {gauge }}^{(A)}(u)=\left[\left(\frac{1}{2 \sinh [z / 2]}\right)^{r_{A}} \cdot \prod_{\alpha \in \Delta_{A}} \frac{\sinh [\alpha(u) / 2]}{\sinh [(\alpha(u)-z) / 2]}\right],
$$

where $r_{A}$ is the rank of the gauge group, $e^{z / 2}=\mathbf{y}$, and $\alpha$ 's are root vectors. A chiral multiplet of charge $q_{I}$, with respect to the Cartan, and $R$-charge $R_{I}$ gives

$$
g_{\text {chiral }}^{(I)}(u)=(-1) \cdot \frac{\sinh \left[\left(q_{I}(u)+\left(R_{I} / 2-1\right) z+f_{I} \cdot a\right) / 2\right]}{\sinh \left[\left(q_{I}(u)+R_{I} z / 2+f_{I} \cdot a\right) / 2\right]},
$$

where $a$ collectively denotes flavor chemical potentials and $f_{I}$ the charges of the chiral multiplet under flavor symmetries.

The space spanned by the Cartan zero modes $u$ is product of cylinders $\left(\mathbb{C}^{*}\right)^{r}$ where $r=\sum_{A} r_{A}$ is the total rank. For quiver theories with the gauge group $\prod_{A} \mathrm{U}\left(d_{A}\right)$, we have $r+1=\sum_{A} d_{A}$ since the overall $\mathrm{U}(1)$ is decoupled. This zero mode space is riddled with singular hypersurfaces defined by poles of $g(u)$, such as $\alpha(u)=z$ and $q_{I}(u)+R_{I} z / 2+f_{I}$. $a=0$, and the Witten index is expressed as sum of iterated residues at co-dimension $r$ singularities. The main technical difficulty is which of such poles contribute and with what residue. Details of this was derived in HKY, to which readers are forwarded, and here we will summarize the result. The result is compactly expressed in terms of Jeffrey-Kirwan residue $[45,47]$ as

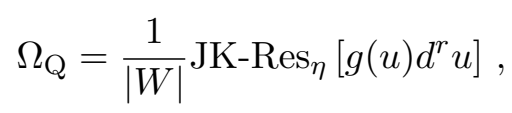

where $W$ is the Weyl group and $\eta$ is an arbitrary but generic vector living in the vector space generated by charges $\{Q\}=\{\alpha\} \cup\{q\}$. The above residue is a summation over all co-dimension $r$ singularities in $\left(\mathbb{C}^{*}\right)^{r}$ that can be defined as the intersection of hyperplanes via a collection of charges $\left\{Q_{i}\right\}$. A singularity where poles due to $r$ charges $\left\{Q_{i_{p}}\right\}$ collide will contribute a term, computed via the JK-residue formula,

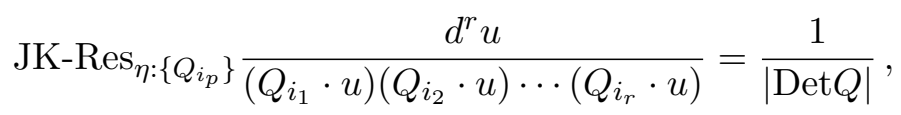

if $\eta$ is a positive linear span of $\left\{Q_{i_{p}}\right\}$; otherwise, JK residue is declared to be zero. We implicitly allowed constant shift of the pole location for notational convenience.

A couple of important points need to be clarified before we can make actual use of this formula. Recall that quiver quantum mechanics, and more generally GLSM quantum mechanics, undergo wall-crossing under continuous change of FI constants. $\Omega_{\mathrm{Q}}$ is therefore a piece-wise constant function of $\zeta$. This aspect is hidden in the fact that, in $\left(\mathbb{C}^{*}\right)^{r}$ spanned by $e^{u}$ 's, there are additional poles located at $\operatorname{Re} u= \pm \infty$ or $\bar{x}_{3}= \pm \infty$. Subtlety in dealing with this additional singularity results in the wall-crossing phenomena. Here we will be content with giving a prescription. The simplest way to achieve this is to assign a charge $Q_{\infty} \equiv-\zeta$ to this asymptotic region ${ }^{5}$ and reject or accept the pole at such places using the

\footnotetext{
${ }^{5} \mathrm{HKY}$ introduced $Q_{\infty}$ in a more limited sense when defining an integrand in the intermediate step, rather than as an effective charge entering the JK test. For this limited use, $Q_{\infty}=\zeta$ chosen there works equally well such that with $\eta=\zeta$, the asymptotic contribution vanish.
} 
same JK residue test with $Q_{\infty}$ as one of $Q_{i}$ 's,

$$
\left\{Q^{\prime}\right\}=\{\alpha\} \cup\{q\} \cup\left\{Q_{\infty}=-\zeta\right\} .
$$

We need to remind ourselves that the hyperplane associated with $Q_{\infty}$ is the asymptotic boundary of $\left(\mathbb{C}^{*}\right)^{r}$. Once this is understood, a natural choice of $\eta$ emerges; if one takes $\eta=\zeta$, where $\zeta$ is now embedded into the charge vector space, the JK positivity test always rejects $Q_{\infty} \equiv-\zeta$, meaning the poles located at the asymptotic region of $\left(\mathbb{C}^{*}\right)^{r}$ can be made to be irrelevant for the Witten index.

However, sometimes this choice is not available because $\zeta$ is not generic, i.e., is spanned by less than $r$ charges. In such cases, we may try to shift $\eta$ slightly away from $\zeta$ but still the asymptotic poles do not contribute. To see how this can be achieved, consider a small deformation $\delta$ such that $\eta=\zeta+\delta$. We wish to see for what choices of $\delta$ the additional charge $Q_{\infty}$ cannot pass the JK positivity test. Suppose it does for some $\delta$ and some collection $\left\{Q_{\infty}, Q_{i_{2}}, \ldots, Q_{i_{r}}\right\}$, i.e.,

$$
\zeta+\delta=b_{1} Q_{\infty}+\sum_{p=2}^{r} b_{i} Q_{i_{p}}
$$

with $b_{1,2, \ldots, r}>0$. This implies

$$
\zeta+\frac{1}{1+b_{1}} \delta=\sum_{p=2}^{r} \frac{b_{p}}{1+b_{1}} Q_{i_{p}},
$$

so that a straight line between $\zeta$ and $\eta$ in the charge vector space encounters a wall spanned by a collection of $r-1$ charges. A rank $r$ charge vector spaces can be divided into chambers by walls which are positive spans of $r-1$ physical charges, which is not to be confused with the physically distinct chambers in the wall-crossing sense defined on the FI constant space. We conclude that as long as $\eta$ lives in the same chamber as $\zeta$ in the charge vector space, the asymptotic pole never enters the JK residue formula. We will be making such choices in all of following computations, and deal only with the hyperplanes associated with the physical charges $\{Q\}=\{\alpha\} \cup\{q\}$.

This naive procedure encounters much difficulties when, at a contributing pole, more than $r$ such hyperplanes meet. For these so-called degenerate cases, the residue computation depends on the order of integration and the contribution from such a point consists of several such iterated residues. This reflects the fact that the middle homology of the Cartan zero mode space at such a singularity is no longer generated by a single cycle and the integral required is a sum of integrals over several such. A couple of constructive procedures are available to deal with such cases, details of which will not be discussed here, as they are available elsewhere $[46,47]$. In this note we follow a constructive procedure of ref. [47], as described by Benini et al. [48].

Finally, we wish to point out that this derivation is performed with finite $\beta$ rather than by taking $\beta \rightarrow \infty$ limit, and can thus potentially fail to capture the true index. This is remedied by taking large $\zeta$ limit while maintaining the chamber [30], which suffices for theories with compact classical moduli space or otherwise by adding enough chemical potential to lift flat directions. There are examples of GLSM for which these remedies are not enough to lift asymptotic flat directions, but this goes beyond the scope of this note. 


\section{Wall-crossing and quiver invariants}

Wall-crossing, which is unique to $1 \mathrm{~d}$ theories, is a discontinuity of supersymmetric spectra in the $\zeta$ space. The co-dimension-one "walls" in the $\zeta$ space are defined as $\sum_{i} n_{i} \zeta_{i}=0$, where we also have $\sum_{i}\left(N_{i}-n_{i}\right) \zeta_{i}=0$. At such places, the phases of central charges of $\Gamma_{1}=\sum_{i} n_{i} \gamma_{i}$ and $\Gamma_{2}=\sum_{i}\left(N_{i}-n_{i}\right) \gamma_{i}$ coincide precisely, and if both $\Gamma_{1,2}$ exist as BPS states, wall-crossing occurs such that degeneracy of $\Gamma=\Gamma_{1}+\Gamma_{2}$ can change suddenly across the wall or at the wall. At the level of equivariant index, this can be phrased as piece-wise constant behavior of $\Omega_{\mathrm{Q}}(\zeta)$ in the space of $\zeta$.

One intuitive way to understand this discontinuity is to consider the so-called "Coulomb" description of the quiver theory, where only the Cartan part of U( $\left.N_{i}\right)$ 's are kept and all other degrees of freedom are integrated out. Naively, this picture is valid when all $\zeta$ 's are small relative to the scale of $1 \mathrm{~d}$ gauge couplings. One ends up with a collection of $\sum N_{i}$ charged particles in $\mathbf{R}^{3}$ space where the vector multiplet scalars live in, and the ground states look like multi-center bound states where individual centers of charge $\gamma_{i}$ 's are balanced against one another by combination of attractive Coulomb-like potential and repulsive "angular momentum" barrier.

The mutual distances of these constituent particles are set by inverses of $\zeta_{i}$ 's, and the wall-crossing discontinuity happens as one or more charged particles, say of total charge $\sum_{i} n_{i} \gamma_{i}$, move off to infinity of $\mathbf{R}^{3}$, relative to the others when $\sum_{i} n_{i} \zeta_{i}$ vanishes. The discontinuity of index occurs because such a state fails square-normalizability. Very general state counting in this picture has been carried out in recent years, which we will denote collectively as

$$
\Omega_{\mathrm{Q}}^{\text {Coulomb }}(\zeta)=\operatorname{Tr}(-1)^{2 J_{3}} \mathbf{y}^{2 I+2 J_{3}} e^{-\beta H_{\mathrm{Q}}^{\text {Coulomb }}(\zeta)}
$$

and which has been compared successfully, for quivers without oriented loops and thus without superpotential, against various mathematical results such as Reineke's results [27] and results deduced from Kontsevich-Soibelman [11] wall-crossing algebra.

However, it turns out that this "Coulomb" picture can miss a huge set of ground states when the quiver admits superpotentials. These additional ground states remain centered and compact near the origin even while $\sum_{i} n_{i} \zeta_{i} \rightarrow 0$, and thus easily survive wall-crossing catastrophe. The quiver invariant can be defined as those states that survive at all the "walls," and they continue to exist as square-normalizable wavefunctions at the intersection of all marginal stability wall. In 1d GLSM, the latter corresponds to the origin of FI constant space, $\zeta_{i}=0$ for all $i$. Counting the index at such a place, one is naturally lead to the definition of the quiver invariant

$$
\left.\Omega_{\mathrm{Q}}\right|_{\text {Inv }}=\lim _{\beta \rightarrow \infty} \operatorname{Tr}_{L^{2}}(-1)^{2 J_{3}} \mathbf{y}^{2 I+2 J_{3}} e^{-\beta H_{\mathrm{Q}}(\zeta=0)} .
$$

Although we will be mostly concerned with quiver theories in this note, it is clear that the same definition can be extended to other 1d GLSM theories, defining GLSM invariants in a similar manner. Note that we took care to impose $L^{2}$ condition on wavefunctions, as $\zeta=0$ generically results in asymptotic runaway directions along the vector multiplet scalars. 
Otherwise, the quantity would be either ill-defined or could give misleading answers. This also tells us direct evaluation will be pretty difficult.

For Abelian cyclic quivers, this split between Coulombic multi-center states and the wall-crossing-safe quiver invariants is clean and has been understood rigorously. For example, let us take triangle quiver with $\left(b_{23}, b_{31}, b_{12}\right)=(4,5,6)$. The quiver admits three different chambers, where the Hodge diamonds turn out to be

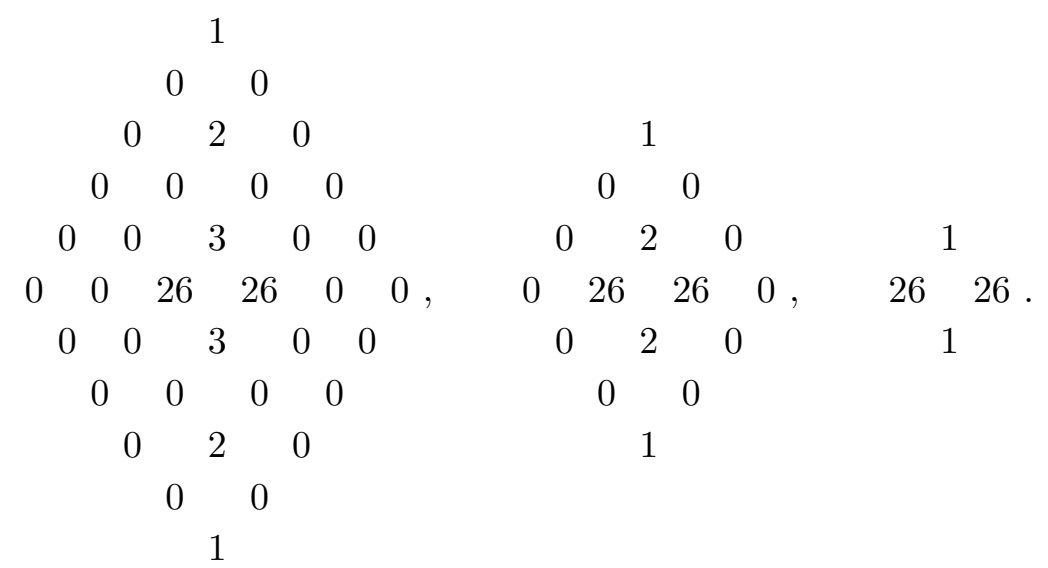

For this simple class, the relevant geometry is entirely toric or a complete intersection therein, so the cohomology is easy to compute.

One important observation, emerged from study of these cyclic Abelian quivers [25, $26,28]$, is that states counted by $\left.\Omega\right|_{\text {Inv }}$ are always $\mathrm{SU}(2)_{R}$ singlet but can be charged under $\mathrm{U}(1)_{R}$, while those counted by $\Omega^{\text {Coulomb }}$ are neutral under $\mathrm{U}(1)_{R}$ and typically in $\mathrm{SU}(2)_{R}$ multiplet: the Coulombic states naturally carry angular momentum in $4 \mathrm{~d}$ sense, since they are always multi-center states, which translates to $\mathrm{SU}(2)_{R}$ charge from the quiver quantum mechanics viewpoint. The fact that they are $\mathrm{U}(1)_{R}$ singlet, a phenomenological observation without a known exception, is related to the so-called "No Exotics Conjecture" [14] that says that no BPS state multiplet of $4 \mathrm{~d} \mathcal{N}=2$ field theory carries $\mathrm{U}(1)_{R}$ charge. On the other hand, $\left.\Omega\right|_{\text {Inv }}$ are undetectable by the Coulombic degrees of freedom only, meaning their wavefunctions are constructed out of the chiral fields and thus effectively spin-less. Such absence of angular momentum for $\left.\Omega\right|_{\text {Inv }}$ states is a generalization of the well-known observation by Ashoke Sen $[42,43]$ that $1 / 4$-BPS and 1/8-BPS black holes of the singlecenter type are angular momentum singlets.

In the low energy nonlinear sigma model limit, $2 J_{3}$ of $\mathrm{SU}(2)_{R}$ and $2 I$ of $\mathrm{U}(1)_{R}$ label the vertical and the horizontal directions of the Hodge diamond. For the above example, $26+26$ states in the horizontal middle belong to $\left.\Omega\right|_{\text {Inv }}$. It turns out that these features of Coulombic states and wall-crossing-safe states being, respectively, vertical and horizontal middle cohomology elements are completely general.

Any wavefunction of multi-center nature will loose its square-normalizability upon $\zeta=0$, among which are states counted by $\Omega_{\mathrm{Q}}^{\text {Coulomb }}(\zeta)$. However, for more general quivers, there are also hybrid type of multi-center states where, among the constituents "particles," one finds quiver invariants of subquivers. Therefore, if one is to study supersymmetric ground states in such multi-center viewpoint, one must count many different kinds of multi- 
center bound states with both elementary constituents and those from quiver invariants of subquivers.

This physically compelling idea has been consolidated into a partition sum identity as follows $[28,29],{ }^{6}$

$$
\Omega_{\mathrm{Q}}(\zeta) \sim \sum_{\mathrm{Q}=\oplus_{p} \mathrm{Q}_{p}} \Omega_{\mathrm{Q} /\left\{\mathrm{Q}_{p}\right\}}^{\text {Coulomb }}\left(\left\{\zeta_{p}\right\}\right) \times\left(\left.\prod_{p} \Omega_{\mathrm{Q}_{p}}\right|_{\text {Inv }}\right) .
$$

The right hand side requires further explanation. ${ }^{7}$ The sum is over all possible partition of the quiver, which is to say all possible partitions of the ranks, $N_{i}=\sum_{p} N_{i}^{(p)}$, with nonnegative integers $N_{i}^{(p)}$ 's. Each such partition defines a set of subquivers $\mathrm{Q}_{p}$ with ranks $N_{i}^{(p)}$. The adjacency matrix $b$ and FI constants $\zeta$ 's of $\mathrm{Q}_{p}$ are the same as those of $\mathrm{Q}$. The quiver denoted as $\mathrm{Q} /\left\{\mathrm{Q}_{p}\right\}$ is an induced quiver where each of subquivers $\mathrm{Q}_{p}$ is treated as if it is a single node of charge $\Gamma_{p}=\sum_{i} N_{i}^{(p)} \gamma_{i}$. The induced adjacency matrix and the induced FI constants of $\mathrm{Q} /\left\{\mathrm{Q}_{p}\right\}$ are determined naturally, e.g., $b_{p q}=\left\langle\Gamma_{p}, \Gamma_{q}\right\rangle$ and $\zeta_{p}=\sum_{i} N_{i}^{(p)} \zeta_{i}$ etc.

The simplest nontrivial example is again the Abelian cyclic quivers, which motivated the above partition sum to begin with. In the latter class, the summation consists of only two terms,

$$
\Omega_{\mathrm{Q}}(\zeta)=1 \times\left.\Omega_{\mathrm{Q}}\right|_{\mathrm{Inv}}+\Omega_{\mathrm{Q}}^{\text {Coulomb }}\left(\left\{\zeta_{i}\right\}\right) \times\left(\prod_{i} 1\right) .
$$

The first term corresponds to $\mathrm{Q}=\mathrm{Q}_{1}$, i.e. $N_{i}^{(1)}=N_{i}=1$, such that $\mathrm{Q} /\left\{\mathrm{Q}_{p}\right\}$ is the trivial single node Abelian quiver. The second corresponds to $\mathrm{Q}=\oplus_{p} \mathrm{Q}_{p}$ with $N_{i}^{(p)}=\delta_{i}^{p}$, so that $\mathrm{Q} /\left\{\mathrm{Q}_{p}\right\}$ is $\mathrm{Q}$ itself. Finally "1" factors are associated with the elementary and free $\mathrm{U}(1)$ quiver, which signals the underlying object, $4 \mathrm{~d}$ quantum state in half-hypermultiplet or the rigidly wrapped D-brane. All other subquivers are tree-like with vanishing $\left.\Omega\right|_{\text {Inv }}$ and are thus absent in the sum. In this class of quivers, states counted by the first spans horizontal middle of the Hodge-decomposed cohomology which remains robust under any of the wall-crossing, while the second spans the vertical middle and changes chamber by chamber. Abelian cyclic quivers are a little special, as states counted by $\Omega_{\mathrm{Q}}^{\text {Coulomb }}$ can be given special geometric meaning [26], via Lefschetz hyperplane theorem, but generalization of this to general quiver is not known.

This partition sum actually goes further than a mere reproduction of true index via multi-center viewpoint. Eq. (4.3), whose idea should extend to the supersymmetric Hilbert space itself, means that one can reconstruct the entire Hodge diamonds, or the entire supersymmetric spectra for any given quiver Q. For general quivers, especially those involving

\footnotetext{
${ }^{6}$ Because this formula originates from a form of Abelianization routine which is natural in Coulombic construction of vacuum states, the precise formula involves various combinatoric factors due to Weyl projections and needs to be phrased via the rational version of the index, $\bar{\Omega}$; here, we refer readers to existing literatures $[16-18]$.

${ }^{7}$ In refs. [28, 29], the counterpart of $\left.\Omega\right|_{\text {Inv }}$ is denoted as $\Omega_{S}$, where $\mathrm{S}$ stands for single-center states. Furthermore, the authors proposed this expansion formulae for Poincaré polynomials rather than for indices, so that $\Omega_{S}$ of theirs is actually an integer rather than Laurent polynomials of $\mathbf{y}$. However, the same expansion formula should work for indices provided that pure Coulombic wavefunctions have vanishing $R$-charges and that all states counted by the quiver invariants are $\mathrm{SU}(2)_{R}$ singlets.
} 
$N_{i}>1$ for some $i$, the cohomology computation is mathematically very challenging. The possibility of a computationally straightforward determination of cohomologies of entire class of quiver varieties is quite remarkable, to say the least.

To illustrate this, take a non-Abelian triangle quiver of ranks $(1,1,3)$ and the adjacency matrix with $\left(b_{23}, b_{31}, b_{12}\right)=(3,5,10)$. Let us denote their indices as $\Omega_{3,5,10}^{1,1,3}$. The relevant quiver invariants are $\left.\Omega_{3,5,10}^{1,1, N}\right|_{\text {Inv }}$ for $N=1,2,3$, as no other subquiver can have a quiver invariant. It turns out that

$$
\begin{aligned}
& \left.\Omega_{3,5,10}^{1,1,1}\right|_{\text {Inv }}=0 \\
& \left.\Omega_{3,5,10}^{1,1,2}\right|_{\text {Inv }}=6 / \mathbf{y}+6 \mathbf{y} \\
& \left.\Omega_{3,5,10}^{1,1,3}\right|_{\text {Inv }}=0
\end{aligned}
$$

Thus, there are only two nontrivial terms in the partition sum;

$$
\Omega_{3,5,10}^{1,1,3}=\left(\Omega_{2}^{1,1}\right)^{\text {Coulomb }} \times\left.\Omega_{3,5,10}^{1,1,2}\right|_{\text {Inv }} \times 1+\left(\Omega_{3,5,10}^{1,1,3}\right)^{\text {Coulomb }} \times 1^{5} .
$$

One is the maximal partition, for which $\mathrm{Q} /\left\{\mathrm{Q}_{p}\right\}=\mathrm{Q}$ itself. The other is $(1,1,3)=$ $(1,1,2) \oplus(0,0,1)$ for which $\mathrm{Q} /\left\{\mathrm{Q}_{p}\right\}$ is a two-node Abelian quiver with the intersection number $\left\langle\gamma_{3}, \gamma_{1}+\gamma_{2}+2 \gamma_{3}\right\rangle=5-3=2$.

For example, in the chamber of the maximal moduli space dimensions, the Witten index is [30]

$$
\Omega_{3,5,10}^{1,1,3}=1 / \mathbf{y}^{6}+2 / \mathbf{y}^{4}-2 / \mathbf{y}^{2}-7-2 \mathbf{y}^{2}+2 \mathbf{y}^{4}+\mathbf{y}^{6},
$$

while the relevant Coulomb indices are

$$
\begin{aligned}
\left(\Omega_{2}^{1,1}\right)^{\text {Coulomb }} & =-1 / \mathbf{y}-\mathbf{y} \\
\left(\Omega_{3,5,10}^{1,1,3}\right)^{\text {Coulomb }} & =1 / \mathbf{y}^{6}+2 / \mathbf{y}^{4}+4 / \mathbf{y}^{2}+5+4 \mathbf{y}^{2}+2 \mathbf{y}^{4}+\mathbf{y}^{6} .
\end{aligned}
$$

From these, we can reconstruct the Hodge diamond

$$
\begin{aligned}
& 1 \\
& \begin{array}{ll}
0 & 0
\end{array} \\
& \begin{array}{lll}
0 & 2 & 0
\end{array} \\
& \begin{array}{llll}
0 & 0 & 0 & 0
\end{array} \\
& \begin{array}{lllll}
0 & 0 & 4 & 0 & 0
\end{array} \\
& \begin{array}{llllll}
0 & 0 & 6 & 6 & 0 & 0
\end{array} \\
& \begin{array}{lllllll}
0 & 0 & 0 & 5 & 0 & 0 & 0
\end{array} \\
& \begin{array}{llllll}
0 & 0 & 6 & 6 & 0 & 0
\end{array} \\
& \begin{array}{lllll}
0 & 0 & 4 & 0 & 0
\end{array} \\
& \begin{array}{llll}
0 & 0 & 0 & 0
\end{array} \\
& \begin{array}{lll}
0 & 2 & 0
\end{array} \\
& \begin{array}{ll}
0 & 0
\end{array} \\
& 1
\end{aligned}
$$

of this chamber. Other chambers can be treated similarly. 
Currently, however, we do not know of any direct and practical computational method for the quiver invariants. In the above example, we actually computed $\Omega_{\mathrm{Q}}$ 's and $\Omega_{\mathrm{Q}}^{\text {Coulomb's }}$ first, and then inferred $\left.\Omega_{\mathrm{Q}}\right|_{\mathrm{Inv}}$ inductively. Note that the Witten index by itself cannot give us the full cohomology information. Construction of the Hodge diamond comes as a bonus along the process. Absence of a direct computational tool for $\left.\Omega_{\mathrm{Q}}\right|_{\text {Inv }}$, despite its very elegant and robust nature, is unsatisfactory. One purpose of this note is to consider how mutation might help us in determining $\Omega_{\mathrm{Q}}$ 's and $\left.\Omega_{\mathrm{Q}}\right|_{\text {Inv }}$ by mapping high-rank quivers to lower-rank ones.

\section{Mutation $\mu_{k}$ on Witten index $\Omega_{\mathrm{Q}}(\zeta)$}

\subsection{Mutations and chambers}

Mutation is a Seiberg-duality [44] on quiver gauge theories, and expected to preserve physics. Depending on dimensions, it works slight differently. In $4 \mathrm{~d}$, a node has the same number of fundamental and anti-fundamental fields, due to gauge anomaly cancelation, so $\mu^{L}=\mu^{R}$. In $2 \mathrm{~d}$, the equality between the incoming arrows and the outgoing arrows is connected to Calabi-Yau condition and no longer necessary for consistency; in principle $\mu^{L}$ and $\mu^{R}$ can induce two different dualities, although Benini et al. [35] argued that, for each mutation step at node $k$, one must choose one of $\mu_{k}^{L, R}$ for which $\mu_{k}\left(N_{k}\right)$ is the larger. In $1 \mathrm{~d}$, even the choice of mutation node is restricted such that, given a point in $\zeta$ space, one could mutate at most two nodes, one by $\mu^{L}$ and the other by $\mu^{R}$.

This happens, for $\mathcal{N}=4$ quiver quantum mechanics, because of wall-crossing phenomena. Given $\zeta$ 's fixed, the mutation is not allowed for all nodes. Physically clean criteria, applied to rank-one Seiberg-Witten theories, were given by Alim et al. [22, 23], who argued that mutation should be thought of as change of basis charges. Here the basis means that the rest of BPS charges can be built as a sum over the basis with non-negative integer coefficients. What classifies a charge as BPS instead of anti-BPS is an arbitrary convention, so by rotating the relevant "upper-half-plane" in the central charge plane, one is sometimes forced to give up a basis element $\gamma_{k}$, in favor of $-\gamma_{k}$. This can affect the rest of basis as well, and $\mu_{k}^{L, R}$ we introduced earlier were proposed to be the correct transformation of basis under such rotation of "upper-half-plane."

Note that this rotation of upper-half-plane mutates one charge at a time, and the choice is not random. The basis element to be mutated has to be the closest to the other lower-half-plane, either along the right-side of the half planes or along the left-side. Thus, one can anticipate that the left(right) mutation will leave physics invariant only when acting on very specific charge. In terms of quiver theories, this translates to inequalities among FI constants; along the real axis of FI constants, we are allowed either to mutate-left the left-most node or to mutate-right the right-most node. For the case of $(1,1, N)$ cyclic triangle quivers, FI constant plane divides into ten subchambers according to which pair of nodes can be mutated left/right as illustrated in figure 2. Each mutation then map each of these subchambers into one chambers of the mutated triangle quiver of type $\left(1,1, N^{\prime}\right)$ as shown in figure 3 . 


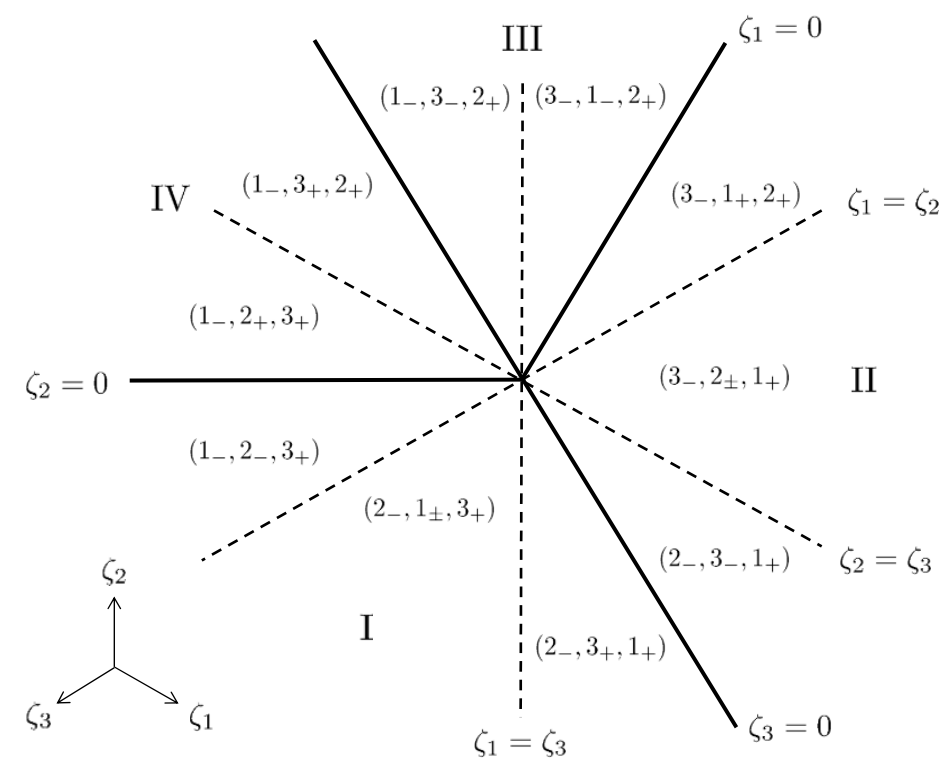

Figure 2. Four physical chambers of $(1,1, N)$ triangle quivers, divided by solid lines. These are further divided into ten sub-chambers by relative ordering of the three FI constants; for example, $\left(2_{-}, 3_{-}, 1_{+}\right)$means $\zeta_{2}<\zeta_{3}<0<\zeta_{1}$. The arrows in the lower-left corner are normal to the respective constant $\zeta$ lines.

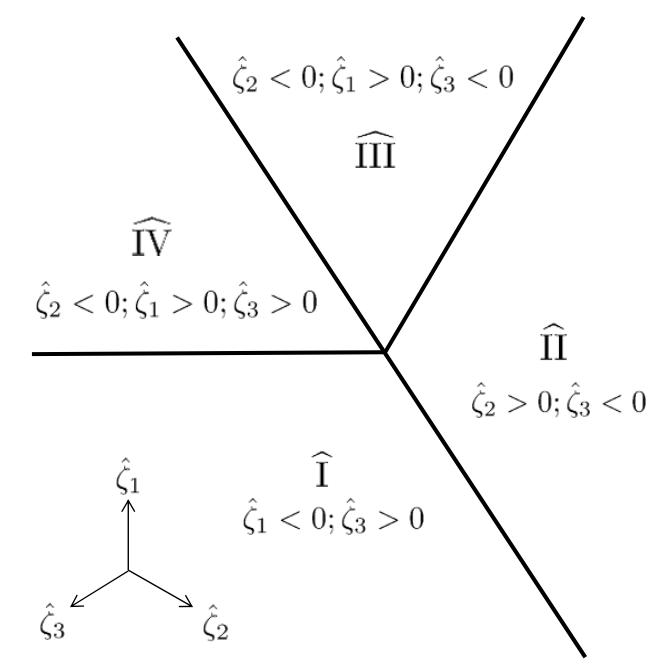

Figure 3. Mutating on node 3 of $(1,1, N)$ quiver brings us back to another $(1,1, \widehat{N})$ quiver. Because the mutation flips arrow orientations, the roles of $\hat{\zeta}_{1}$ and $\hat{\zeta}_{2}$ are exchanged relative to those of $\zeta_{1,2}$. The left mutation, allowed in three sub-chambers of figure 2 with most negative $\zeta_{3}$, maps indices of chambers II and III, respectively, to those of chambers $\widehat{I V}$ and $\widehat{\mathrm{I}}$. Similarly, the right mutation, allowed in three sub-chambers of figure 2 with most positive $\zeta_{3}$, maps indices of chambers I and IV, respectively, to those of chambers $\widehat{\text { III }}$ and $\widehat{\text { II. }}$ 
One immediate question to be asked here is what happens if $\mu_{k}\left(N_{k}\right)$ happen to be negative for some $k$. The mutation is ill-defined because a negative rank appears in the node $k$ of $\widehat{Q}$. Does this simply mean that the mutation map become unavailable? Or could there be still additional information about the original quiver? Let us observe that the index of the original quiver vanishes whenever $\gamma_{k}$ is the left-most (right-most) and $\mu_{k}^{L}\left(N_{k}\right)<0\left(\mu_{k}^{R}\left(N_{k}\right)<0\right)$, which follows from the $D$-term condition at node $k$,

$$
X_{k} X_{k}^{\dagger}-Y_{k}^{\dagger} Y_{k}=\zeta_{k} I_{N_{k} \times N_{k}}
$$

where $X_{k}$ is a rectangular complex matrix of $N_{k} \times\left(\sum_{j}\left[b_{j k}\right]_{+} N_{j}\right)$ type and collectively denotes all chiral multiplets associated with incoming arrows. $Y_{k}$ is of type $\left(\sum_{j}\left[b_{k j}\right]_{+} N_{j}\right) \times$ $N_{k}$ and represents the collection of all outgoing arrows. When $\zeta_{k}$ is negative (positive), the right hand side is of rank $N_{k}$ with all negative (positive) and equal eigenvalues, and this $D$-term equation can be solved only if $Y(X)$ is of rank $N_{k}$ also. When $\zeta_{k}$ is the left-most (right-most) and thus necessarily negative (positive), this condition for non-empty moduli space translates, upon the respective mutation, to $\mu_{k}^{L}\left(N_{k}\right) \geq 0\left(\mu_{k}^{R}\left(N_{k}\right) \geq 0\right)$. Therefore we conclude that whenever a formally valid mutation results in a negative rank of the mutated node, the original quiver must have been in a physically empty chamber with a vanishing Witten index. In this sense, it suffices to consider the original quivers and the chambers thereof such that allowed mutation results in $\mu_{k}\left(N_{k}\right) \geq 0$, to which cases we will restrict ourselves.

With the index counting enabled by HKY's general formula, we wish to test this mutation idea explicitly by applying to a simplest class of triangle quivers. We will perform numerical test as well as illustrate how HKY formula itself exhibits invariance under such mutations. The latter may be generalized to a larger class of quivers, establishing the mutation invariance rigorously at the level of index theorem.

\subsection{A numerical check and a subtlety}

Before we plunge into more analytical demonstration in next subsection, let us briefly check the validity of the mutation invariance with a particular example of triangle quivers with ranks $(1,1,2)$ and the intersection numbers $(4,5,7)$ of figure 4 . This will serve to check the aforementioned assertion, regarding invariance of Witten indices of particular chambers as well as non-preservation of Witten indices of "wrong" chambers. Indices of the original quiver were computed in ref. [30],

$$
\begin{aligned}
\Omega(\mathrm{I}) & =50 \\
\Omega(\mathrm{II}) & =1 / \mathbf{y}^{4}+2 / \mathbf{y}^{2}+87+2 \mathbf{y}^{2}+\mathbf{y}^{4}, \\
\Omega(\mathrm{III}) & =1 / \mathbf{y}^{6}+2 / \mathbf{y}^{4}+4 / \mathbf{y}^{2}+89+4 \mathbf{y}^{2}+2 \mathbf{y}^{4}+\mathbf{y}^{6}, \\
\Omega(\mathrm{IV}) & =1 / \mathbf{y}^{6}+2 / \mathbf{y}^{4}+4 / \mathbf{y}^{2}+54+4 \mathbf{y}^{2}+2 \mathbf{y}^{4}+\mathbf{y}^{6} .
\end{aligned}
$$

Under the left mutation, we find a quiver with ranks $(1,1,3)$ and intersection numbers $(-4,-5,-13)$ with indices,

$$
\Omega(\widehat{\mathrm{I}})=1 / \mathbf{y}^{6}+2 / \mathbf{y}^{4}+4 / \mathbf{y}^{2}+89+4 \mathbf{y}^{2}+2 \mathbf{y}^{4}+\mathbf{y}^{6},
$$



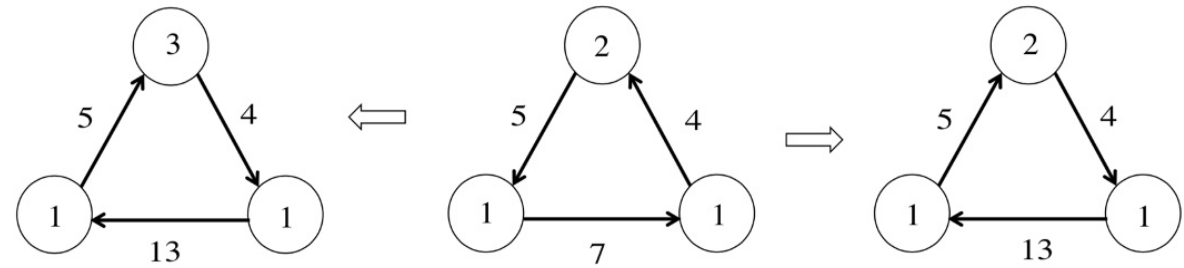

Figure 4. An explicit example of mutation. Witten indices are computed for all four chambers for each of the three quivers, showing that mutation selection rule is necessary.

$$
\begin{aligned}
\Omega(\widehat{\mathrm{II}}) & =35, \\
\Omega(\widehat{\mathrm{III}}) & =1 / \mathbf{y}^{4}+2 / \mathbf{y}^{2}+37+2 \mathbf{y}^{2}+\mathbf{y}^{4}, \\
\Omega(\widehat{\mathrm{IV}}) & =1 / \mathbf{y}^{4}+2 / \mathbf{y}^{2}+87+2 \mathbf{y}^{2}+\mathbf{y}^{4} .
\end{aligned}
$$

Note that $\Omega(\mathrm{II})=\Omega(\widehat{\mathrm{IV}})$ and $\Omega(\mathrm{III})=\Omega(\widehat{\mathrm{I}})$, as anticipated. Under the right mutation, we find a quiver with ranks $(1,1,2)$ and intersection numbers $(-4,-5,-13)$ with indices,

$$
\begin{aligned}
\Omega(\widehat{\mathrm{I}})= & 1 / \mathbf{y}^{10}+2 / \mathbf{y}^{8}+4 / \mathbf{y}^{6}+6 / \mathbf{y}^{4}+8 / \mathbf{y}^{2} \\
& +58+8 \mathbf{y}^{2}+6 \mathbf{y}^{4}+4 \mathbf{y}^{6}+2 \mathbf{y}^{8}+\mathbf{y}^{10}, \\
\Omega(\widehat{\mathrm{II}})= & 1 / \mathbf{y}^{6}+2 / \mathbf{y}^{4}+4 / \mathbf{y}^{2}+54+4 \mathbf{y}^{2}+2 \mathbf{y}^{4}+\mathbf{y}^{6}, \\
\Omega(\widehat{\mathrm{III}})= & 50, \\
\Omega(\widehat{\mathrm{IV}})= & 50 .
\end{aligned}
$$

We find that $\Omega(\mathrm{I})=\Omega(\widehat{\mathrm{III}})$ and $\Omega(\mathrm{IV})=\Omega(\widehat{\mathrm{II}})$, again as anticipated.

Perhaps equally noteworthy is the fact that if one starts in disallowed sub-chambers, where mutation on this node is not justified, Witten indices before and after the mutation do not match. In fact, even the dimension of the classical moduli spaces can differ before and after mutation. This example thus demonstrates that the selection rules for the mutable node and choice of the mutation orientation are very much necessary.

Apart from checking the mutation invariance numerically, this exercise gives a valuable hint on how to demonstrate mutation invariance between a pair of $(1,1, N)$ type quivers. For general quivers, classifying poles according to JK positivity test poses a big combinatorial challenge. This is further aggravated by the presence of degenerate poles where more than $r$ singular hyperplanes collide. When such a degenerate pole passes JK positivity test, the iterated residue becomes order-dependent and further combinatorial task emerges. Such technical issues, however, are much ameliorated when one can exclude hyperplanes associated with vector multiplets from the analysis. This not only reduces poles passing JK test drastically but also tends to remove a lot of degenerate singularities.

For simple quivers, such as primitive tree-like quivers, there is a reasonable argument why JK-acceptable singularities involving the vector multiplet poles must have a vanishing residue [48]. This follows from a counting of the net number of zeros against the net number of poles. For other quivers, such as our triangles with a loop, this argument does not extend straightforwardly. For example, the pole due to the chirals between nodes 1 and 
2 can coincide with a vector multiplet pole of node 3 , such that the vanishing argument due to counting of zeros and poles no longer works. Furthermore, singularities of this type tend to fail the so-called projective property which enables one to derive the residue formulae.

In the end, however, extensive numerical exercises with $(1,1, N)$ quivers lead us to believe that the vector multiplet poles need not be considered at all for this class of quivers. ${ }^{8}$ Most of the singularities involving vector multiplet poles and also passing JK positivity test, can be seen to have a vanishing residue straight-forwardly. The main issue is how to deal with those non-projective singularities. We have regulated these by shifting the coordinates to split them artificially to projective ones, evaluate the residues, and "unshift." The reduced projective singularities give a vanishing residue, again due to the vector multiplet poles being canceled by chiral zeros, and we are back to the statement that vector multiplet poles need not be considered. This simplifies the problem enormously since for each chamber there is exactly one iterated residue integral that contributes to the index. Establishing duality between a mutation pair of $(1,1, N)$ quivers amounts to showing these two residues agree with each other regardless of the intersection numbers $(b, c, a)$ and $(-b,-c, a-b c)$, which we will show in the next subsection.

\subsection{Mutation invariance of Witten index}

The prototype of mutation invariance for 1d GLSM can be found in SQCD-like theories with a single $\mathrm{U}\left(N_{c}\right)$ gauge group coupled to $N_{f}$ and $N_{a}$ number of fundamental and antifundamental chirals, as drawn in the middle of figure 5 . The one-loop determinant of this theory is

$$
\begin{aligned}
g(u, z)= & \left(\frac{1}{2 \sinh [z / 2]}\right)^{N_{c}} \prod_{i \neq j} \frac{\sinh \left[\left(u_{i}-u_{j}\right) / 2\right]}{\sinh \left[\left(u_{i}-u_{j}-z\right) / 2\right]} \\
& \times \prod_{i=1}^{N_{c}} \prod_{\alpha=1}^{N_{f}} \frac{-\sinh \left[\left(u_{i}-a_{\alpha}+R_{f} z / 2-z\right) / 2\right]}{\sinh \left[\left(u_{i}-a_{\alpha}+R_{f} z / 2\right) / 2\right]} \\
& \times \prod_{i=1}^{N_{c}} \prod_{\beta=1}^{N_{a}} \frac{-\sinh \left[\left(-u_{i}+b_{\beta}+R_{a} z / 2-z\right) / 2\right]}{\sinh \left[\left(-u_{i}+b_{\beta}+R_{a} z / 2\right) / 2\right]},
\end{aligned}
$$

where $a_{\alpha}$ 's and $b_{\beta}$ 's are flavor fugacities. Although $R$-charges in this simple theory are ambiguous due to possible mixing with flavor and gauge charges, we keep them explicitly as we wish to embed the theory to a larger theory later on.

This theory has two chambers distinguished by sign of $\zeta$. The index at $\zeta>0$ can be

\footnotetext{
${ }^{8}$ Irrelevance of vector multiplet poles is hardly a general statement. Counterexamples include nonprimitive Kronecker quivers as well as, more obviously, $\mathrm{SU}(n)$ gauged quantum mechanics without matter multiplets. Note that these examples have flat Coulombic directions, however, so the quantity computed by HKY is not true $L^{2}$ index. Nevertheless, these examples shows that the existing prescription rules cannot by themselves preclude vector multiplet poles. Establishing general criteria on when we are allowed to ignore vector multiplet poles will go a long way for our understanding of the Witten index of general GLSM.
} 


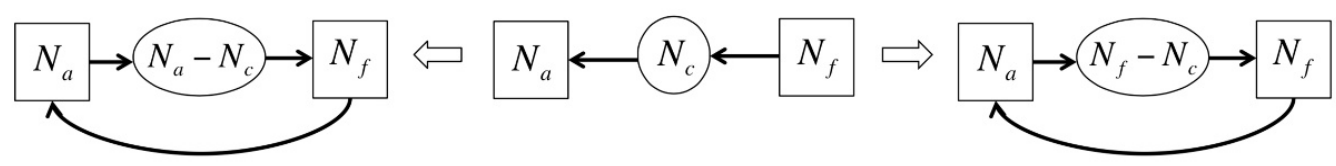

Figure 5. Seiberg-like dualities for 1d SQCD. There exist two different duality maps depending on sign of the FI parameter.

evaluated by sum over all possible configurations of picking up $N_{c}$ poles in the fundamentals,

$$
\begin{aligned}
\operatorname{JK}^{-r}{ }_{\zeta>0} g(u, z)= & \sum_{A \in C\left(N_{f}, N_{c}\right)} \prod_{\substack{i \in A \\
j \in A^{\prime}}} \frac{-\sinh \left[\left(a_{i}-a_{j}-z\right) / 2\right]}{\sinh \left[\left(a_{i}-a_{j}\right) / 2\right]} \\
& \times \prod_{i \in A} \prod_{\beta=1}^{N_{a}} \frac{-\sinh \left[\left(-a_{i}+b_{\beta}+\left(R_{f}+R_{a}\right) z / 2-z\right) / 2\right]}{\sinh \left[\left(-a_{i}+b_{\beta}+\left(R_{f}+R_{a}\right) z / 2\right) / 2\right]},
\end{aligned}
$$

where summation is taken over $\left(\begin{array}{l}N_{f} \\ N_{c}\end{array}\right)$ choices of a set $A$ which choose $N_{c}$ fugacities of fundamentals. We also denote $A^{\prime}$ by its complement. Note that we can rewrite this expression as

$$
\begin{aligned}
\sum_{A^{\prime} \in C\left(N_{f}, N_{f}-N_{c}\right)} & \prod_{\substack{i \in A \\
j \in A^{\prime}}} \frac{-\sinh \left[\left(-a_{j}+a_{i}-z\right) / 2\right]}{\sinh \left[\left(-a_{j}+a_{i}\right) / 2\right]} \\
\times & \prod_{j \in A^{\prime}} \prod_{\beta=1}^{N_{a}} \frac{-\sinh \left[\left(a_{j}-b_{\beta}-\left(R_{a}+R_{f}\right) z / 2\right) / 2\right]}{\sinh \left[\left(a_{j}-b_{\beta}+\left(2-R_{a}-R_{f}\right) z / 2\right) / 2\right]} \\
\times & \prod_{\alpha \in A \cup A^{\prime}} \prod_{\beta=1}^{N_{a}} \frac{-\sinh \left[\left(-a_{\alpha}+b_{\beta}+\left(R_{a}+R_{f}\right) z / 2-z\right) / 2\right]}{\sinh \left[\left(-a_{\alpha}+b_{\beta}+\left(R_{a}+R_{f}\right) z / 2\right) / 2\right]}
\end{aligned}
$$

which is nothing but the index of the theory with $\mathrm{U}\left(N_{f}-N_{c}\right)$ gauge group with same number of (anti-)fundamentals together with $N_{f} N_{a}$ mesons by superpotential $W=\operatorname{Tr} \bar{\Phi} M \Phi$. Especially, the theory is mapped to the chamber with $\zeta^{\prime}<0$ of the dual theory. Note that the $R$-charges of the dual quiver is shifted to $\left(R_{f}^{\prime}, R_{a}^{\prime}, R_{a}+R_{f}\right)$ where $R_{f}^{\prime}+R_{a}^{\prime}=2-R_{f}-R_{a}$.

This shows that $1 \mathrm{~d} \mathcal{N}=4$ SQCD theories also exhibit Seiberg-like duality which is very similar to that of $2 \mathrm{~d}, 3 \mathrm{~d}$ and $4 \mathrm{~d}[35,36,44]$ with the same amount of supersymmetries. Of course, somewhat special feature of $1 \mathrm{~d}$ version is that the theory experiences wall-crossing so that the duality map changes when we go to $\zeta<0$ chamber of the original theory. At this chamber, the JK-residue picks all poles from anti-fundamentals, whose index is similarly mapped to the theory with $\zeta^{\prime \prime}>0$ chamber of $\mathrm{U}\left(N_{a}-N_{c}\right)$ gauge group. For this example, this selection merely tells us whether $\mu^{L}$ or $\mu^{R}$ is the right mutation to perform.

The two types of dualities described above can be thought of as prototypes of the right and left mutation of the quiver quantum mechanics respectively. One might expect that the mutation invariance for general quivers can be straightforwardly proven by gauging the flavor nodes of 1d SQCD example, but this procedure cannot be easily justified when degenerate singularities appear. For such a case, the JK-residue description requires particular order of taking residue integral, which makes it illegal to integrate out Cartans of the 

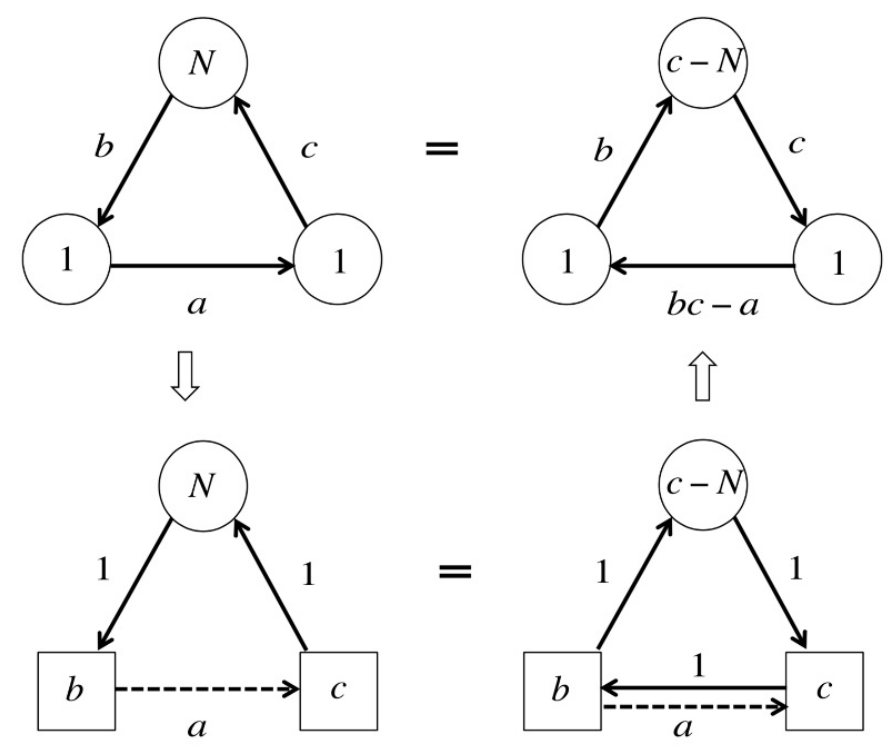

Figure 6. A diagrammatic proof of mutation invariance under the right mutation $\mu^{R}$ on node 3 for $(1,1, N)$ quivers when $\zeta_{3}$ is right-most. The down arrow corresponds replacing two Abelian nodes as $b$ and $c$ flavor nodes, with all chemical potentials turned on. Dashed arrows, which are actually singlets under $\mathrm{SU}(b) \times \mathrm{SU}(c)$, are the original bifundamentals between node 1 and 2 , and does not participate the mutation process. They should be understood as singlet under $b$ and $c$ flavor nodes. The up arrow is a reverse process of turning off the chemical potentials and gauging the overall U(1) in each node. The equality between to the two bottom quivers follows from the SQCD mutation.

mutating node prior to that of the flavorized nodes. Despite these subtleties, there exist some classes of non-Abelian examples that we can prove the mutation equivalence based on SQCD example. Consider quivers with dimension vector $(1,1, N)$ and their sub-chambers where $\zeta_{3}$ is right-most among $\zeta^{\prime}$ 's. This corresponds to the three sub-chambers in figure 2, which belongs to parts of I and IV physical chamber. Figure 6 illustrates roughly how the proof of mutation invariance goes.

First of all, with respect to the Cartan directions, $\left\{e_{0} ; e_{1}, \cdots, e_{N}\right\}$, let us denote the charges of three bifundamentals as

$$
Q_{X}=e_{0}, \quad Q_{Y_{i}}=e_{i}, \quad Q_{Z_{i}}=-e_{0}-e_{i},
$$

and assign the $R$-charges by $\left(R_{X}, R_{Y}, R_{Z}\right)=(0,0,2)$ respectively, which is consistent with a cubic superpotential of type $X Y Z$. Recall that, since only $R$-charge information enters the Witten index, genericity of the superpotential consistent with $\mathrm{U}(1)_{R}$ and the gauge symmetry is implicitly assumed. Then the one-loop determinant of $(1,1, N)$ quiver is given by [30]

$$
\begin{aligned}
g & =\left(\frac{1}{2 \sinh [z / 2]}\right)^{N+1} \prod_{i \neq j} \frac{\sinh \left[\left(u_{i}-u_{j}\right) / 2\right]}{\sinh \left[\left(u_{i}-u_{j}-z\right) / 2\right]} \times\left(\frac{-\sinh \left[\left(u_{0}-z\right) / 2\right]}{\sinh \left[u_{0} / 2\right]}\right)^{a} \\
& \times \prod_{i=1}^{N}\left(\frac{-\sinh \left[\left(u_{i}-z\right) / 2\right]}{\sinh \left[u_{i} / 2\right]}\right)^{c} \prod_{i=1}^{N}\left(\frac{-\sinh \left[\left(-u_{0}-u_{i}\right) / 2\right]}{\sinh \left[\left(-u_{0}-u_{i}+z\right) / 2\right]}\right)^{b} .
\end{aligned}
$$


If we put the $\eta$ parameter as

$$
\eta=\zeta+\epsilon\left(N e_{1}+(N-1) e_{2}+\cdots+2 e_{N-1}+e_{N}\right),
$$

with sufficiently small $\epsilon$ so that $\zeta$ and $\eta$ are in the same chamber in the space of charge vectors.

The JK-residue formula at each chamber reads as follow. In chamber I, the index gets contribution from poles of $X$ and $Y_{i}$ 's, where we have

$$
\Omega(\mathrm{I})=\frac{1}{N !} \operatorname{res}_{u_{0}=0} \operatorname{res}_{u_{N}=0} \cdots \operatorname{res}_{u_{1}=0} g(u, z) .
$$

In chamber II, since $\eta$ is in a positive cone of $X$ and $Z_{i}$ 's, we have

$$
\Omega(\mathrm{II})=\frac{(-1)^{N}}{N !} \operatorname{res}_{u_{0}=0} \operatorname{res}_{u_{N}=z-u_{0}} \cdots \operatorname{res}_{u_{1}=z-u_{0}} g(u, z) .
$$

On the other hand, at chamber III and IV where $Y_{i}$ 's and $Z_{i}$ 's contribute, the singularity is degenerate. A single ordered charge set contributes to the integral at each chamber, which reads, for chamber III,

$$
\Omega(\mathrm{III})=\frac{(-1)^{N+1}}{N !} \operatorname{res}_{u_{0}=z} \operatorname{res}_{u_{N}=z-u_{0}} \cdots \operatorname{res}_{u_{1}=z-u_{0}} g(u, z),
$$

and for chamber IV, we have

$$
\Omega(\mathrm{IV})=\frac{-1}{N !} \operatorname{res}_{u_{0}=z} \operatorname{res}_{u_{N}=0} \cdots \operatorname{res}_{u_{1}=0} g(u, z) .
$$

Note that the order of taking residue is crucial for the latter two cases.

Now, let us define new functions $G_{1}\left(u_{0}\right)$ and $G_{2}\left(u_{0}\right)$ as follows

$$
G_{1}\left(u_{0}\right):=\frac{1}{N !} \operatorname{res}_{u_{N}=0} \cdots \operatorname{res}_{u_{1}=0} g(u, z),
$$

and

$$
G_{2}\left(u_{0}\right):=\frac{(-1)^{N}}{N !} \operatorname{res}_{u_{N}=z-u_{0}} \cdots \operatorname{res}_{u_{1}=z-u_{0}} g(u, z) .
$$

Then For chamber I and IV, the index is expressed as

$$
\Omega(\mathrm{I})=\operatorname{res}_{u_{0}=0} G_{1}\left(u_{0}\right), \text { and } \Omega(\mathrm{IV})=-\operatorname{res}_{u_{0}=z} G_{1}\left(u_{0}\right),
$$

while for chamber II and III, we have

$$
\Omega(\mathrm{II})=\operatorname{res}_{u_{0}=0} G_{2}\left(u_{0}\right), \text { and } \Omega(\mathrm{III})=-\operatorname{res}_{u_{0}=z} G_{2}\left(u_{0}\right) .
$$

Meanwhile, for the dual quiver with ranks $(1,1, c-N)$ and intersection numbers $(-b,-c,-b c+a)$, we similarly have

$$
\begin{aligned}
g_{\text {dual }}= & \left(\frac{1}{2 \sinh [z / 2]}\right)^{c-N+1} \prod_{a \neq b} \frac{\sinh \left[\left(v_{a}-v_{b}\right) / 2\right]}{\sinh \left[\left(v_{a}-v_{b}-z\right) / 2\right]} \times\left(\frac{-\sinh \left[\left(-v_{0}-z\right) / 2\right]}{\sinh \left[-v_{0} / 2\right]}\right)^{b c-a} \\
& \times \prod_{a=1}^{c-N}\left(\frac{-\sinh \left[\left(-v_{a}-z\right) / 2\right]}{\sinh \left[-v_{a} / 2\right]}\right)^{c} \prod_{a=1}^{c-N}\left(\frac{-\sinh \left[\left(v_{0}+v_{a}\right) / 2\right]}{\sinh \left[\left(v_{0}+v_{a}+z\right) / 2\right]}\right)^{b}
\end{aligned}
$$


and the indices for the four chambers can be written as

$$
\begin{aligned}
& \Omega(\widehat{\mathrm{I}})=-\operatorname{res}_{v_{0}=0} \widehat{G}_{1}\left(v_{0}\right), \quad \Omega(\widehat{\mathrm{IV}})=\operatorname{res}_{v_{0}=-z} \widehat{G}_{1}\left(v_{0}\right) \\
& \Omega(\widehat{\mathrm{II}})=-\operatorname{res}_{v_{0}=0} \widehat{G}_{2}\left(v_{0}\right), \quad \Omega(\widehat{\mathrm{III}})=\operatorname{res}_{v_{0}=-z} \widehat{G}_{2}\left(v_{0}\right) \text {, }
\end{aligned}
$$

where

$$
\begin{aligned}
& \widehat{G}_{1}\left(v_{0}\right)=\frac{(-1)^{c-N}}{(c-N) !} \operatorname{res}_{v_{c-N}=0} \cdots \operatorname{res}_{v_{1}=0} g_{\text {dual }}\left(v_{i}, v_{0}\right), \\
& \widehat{G}_{2}\left(v_{0}\right)=\frac{1}{(c-N) !} \operatorname{res}_{v_{c-N}=-v_{0}-z} \cdots \operatorname{res}_{v_{1}=-v_{0}-z} g_{\text {dual }}\left(v_{i}, v_{0}\right) .
\end{aligned}
$$

In order to prove the equivalence of the indices under the right mutation of the node 3 , we show below that

$$
G_{1}\left(u_{0}\right)=\widehat{G}_{2}\left(u_{0}-z\right)
$$

holds, from which it would follow that

$$
\Omega(\mathrm{I})=\Omega(\widehat{\mathrm{III}}), \quad \Omega(\mathrm{IV})=\Omega(\widehat{\mathrm{II}}),
$$

where $R$-charges for the dual theory are now assigned as $\left(R_{\widehat{X}}, R_{\widehat{Y}}, R_{\widehat{Z}}\right)=(2,0,0)$.

For this purpose, we introduce auxiliary variables $a_{\gamma=1, \cdots c}$ to split the order $c$ pole defined by $u_{i}=0$ into sum over residues over various simple poles;

$$
\begin{aligned}
G_{1}\left(u_{0}\right) & =\frac{1}{N !} \operatorname{res}_{u_{N}=0} \cdots \operatorname{res}_{u_{1}=0} g(u, z) \\
& =\frac{1}{N !} \operatorname{res}_{u_{N}=0} \cdots \operatorname{res}_{u_{1}=0} \lim _{a_{\gamma} \rightarrow 0} \tilde{g}\left(u, z, a_{\gamma}\right) \\
& =\frac{1}{N !} \lim _{a_{\gamma} \rightarrow 0} \sum_{\tau} \operatorname{res}_{u_{N}=a_{\tau(N)}} \cdots \operatorname{res}_{u_{1}=a_{\tau(1)}} \tilde{g}\left(u, z, a_{\gamma}\right),
\end{aligned}
$$

where $\tilde{g}\left(u, z, a_{\gamma}\right)$ is defined by

$$
\begin{aligned}
\tilde{g}\left(u, z, a_{\gamma}\right)= & \left(\frac{1}{2 \sinh [z / 2]}\right)^{N+1} \prod_{i \neq j} \frac{\sinh \left[\left(u_{i}-u_{j}\right) / 2\right]}{\sinh \left[\left(u_{i}-u_{j}-z\right) / 2\right]} \times\left(\frac{-\sinh \left[\left(u_{0}-z\right) / 2\right]}{\sinh \left[u_{0} / 2\right]}\right)^{a} \\
& \times \prod_{i=1}^{N} \prod_{\gamma=1}^{c} \frac{-\sinh \left[\left(u_{i}-a_{\gamma}-z\right) / 2\right]}{\sinh \left[\left(u_{i}-a_{\gamma}\right) / 2\right]} \prod_{i=1}^{N}\left(\frac{-\sinh \left[\left(-u_{0}-u_{i}\right) / 2\right]}{\sinh \left[\left(-u_{0}-u_{i}+z\right) / 2\right]}\right)^{b} \cdot
\end{aligned}
$$

In the last line of (5.24), the summation is taken over all different $c^{N}$ choices of $\tau(i)$ for each Cartan $u_{i}$. The evaluation of the residue integral for $G_{1}\left(u_{0}\right)$ becomes,

$$
\begin{gathered}
G_{1}\left(u_{0}\right)=\frac{1}{N !} \lim _{a_{\gamma} \rightarrow 0} \sum_{\tau} \operatorname{res}_{u_{N}=a_{\tau(N)}} \cdots \operatorname{res}_{u_{1}=a_{\tau(1)}} \tilde{g}\left(u, z, a_{\gamma}\right) \\
=\lim _{a_{\gamma} \rightarrow 0} \frac{1}{2 \sinh [z / 2]} \sum_{C(c, N)} \prod_{\substack{\gamma \in A \\
\gamma^{\prime} \in A^{\prime}}} \frac{-\sinh \left[\left(a_{\gamma}-a_{\gamma^{\prime}}-z\right) / 2\right]}{\sinh \left[\left(a_{\gamma}-a_{\gamma^{\prime}}\right) / 2\right]}\left(\frac{-\sinh \left[\left(u_{0}-z\right) / 2\right]}{\sinh \left[u_{0} / 2\right]}\right)^{a} \\
\times \prod_{\gamma \in A}\left(\frac{-\sinh \left[\left(-u_{0}-a_{\gamma}\right) / 2\right]}{\sinh \left[\left(-u_{0}-a_{\gamma}+z\right) / 2\right]}\right)^{b}
\end{gathered}
$$


where $C(c, N)$ denotes all combinations of subset $A=\left\{\gamma_{i} \mid u_{i}=a_{\gamma_{i}}, i=1, \cdots N\right.$ such that $a_{\gamma_{i}} \neq a_{\gamma_{j}}$ for all $\left.i \neq j\right\}$, and $A^{\prime}$ is complement of a set $A$. Furthermore, using

$$
\begin{aligned}
& \left(\frac{-\sinh \left[\left(u_{0}-z\right) / 2\right]}{\sinh \left[u_{0} / 2\right]}\right)^{a} \\
& =\lim _{a_{\gamma} \rightarrow 0}\left(\frac{-\sinh \left[-u_{0} / 2\right]}{\sinh \left[\left(-u_{0}+z\right) / 2\right]}\right)^{b c-a}\left(\prod_{\gamma \in A \cup A^{\prime}} \frac{-\sinh \left[\left(-u_{0}-a_{\gamma}+z\right) / 2\right]}{\sinh \left[\left(-u_{0}-a_{\gamma}\right) / 2\right]}\right)^{b},
\end{aligned}
$$

we can alternatively express each term in the sum of (5.26) as

$$
\begin{aligned}
& \prod_{\substack{\gamma \in A \\
\gamma^{\prime} \in A^{\prime}}} \frac{-\sinh \left[\left(a_{\gamma}-a_{\gamma^{\prime}}-z\right) / 2\right]}{\sinh \left[\left(a_{\gamma}-a_{\gamma^{\prime}}\right) / 2\right]} \\
& \times\left(\frac{-\sinh \left[-u_{0} / 2\right]}{\sinh \left[\left(-u_{0}+z\right) / 2\right]}\right)^{b c-a}\left(\prod_{\gamma \in A^{\prime}} \frac{-\sinh \left[\left(-u_{0}-a_{\gamma}+z\right) / 2\right]}{\sinh \left[\left(-u_{0}-a_{\gamma}\right) / 2\right]}\right)^{b},
\end{aligned}
$$

which is exactly equal to a term in $\widehat{G}_{2}\left(u_{0}-z\right)$.

Although the limit $a_{\gamma} \rightarrow 0$ is not well-defined for individual terms, it can be shown that the limit gives finite answer when we sum up all combinations $C(c, N)$. After we add up all terms in the summation, (5.26) can be written in a following form.

$$
\frac{f\left(A_{\gamma}, U_{0}\right)}{\prod_{\gamma<\delta}\left(A_{\gamma}-A_{\delta}\right) \prod_{\text {all } \gamma}\left(A_{\gamma}-U_{0}\right)},
$$

where $U_{0}=e^{u_{0} / 2}, A_{\gamma}=e^{a_{\gamma} / 2}$, and $f\left(A_{\gamma}, U_{0}\right)$ is an anti-symmetric polynomial in $A_{\gamma}$ 's. Since every antisymmetric polynomial is divisible by the Vandermonde determinant to a symmetric polynomial, the first factor in the denominator is always canceled and $a_{\gamma} \rightarrow 0$ limit is well-defined at the generic value of $U_{0}$. This gives

$$
\begin{array}{rlrl}
\Omega(\mathrm{I}) & =\Omega(\widehat{\mathrm{III}}), & & \text { for region }\left(2_{-}, 1_{ \pm}, 3_{+}\right) \text {and }\left(1_{-}, 2_{-}, 3_{+}\right) \\
\Omega(\mathrm{IV}) & =\Omega(\widehat{\mathrm{II}}), & \text { for region }\left(1_{-}, 2_{+}, 3_{+}\right)
\end{array}
$$

under $\mu_{3}^{R}$ as promised, where each regions of original FI parameters are drawn in figure 2 .

The left-mutation $\mu_{3}^{L}$ on node 3 when $\zeta_{3}$ is left-most can be checked similarly, resulting in the identities

$$
\begin{aligned}
\Omega(\mathrm{II}) & =\Omega(\widehat{\mathrm{IV}}), & \text { for region }\left(3_{-}, 1_{+}, 2_{+}\right) \text {and }\left(3_{-}, 2_{ \pm}, 1_{+}\right) \\
\Omega(\mathrm{III}) & =\Omega(\widehat{\mathrm{I}}), & \text { for region }\left(3_{-}, 1_{-}, 2_{+}\right)
\end{aligned}
$$

where we remind readers that the mutated quiver under $\mu_{3}^{L}$ is not the same as the mutated quiver under $\mu_{3}^{R}$ unless $b=c$.

Let us briefly comment on what happens for the mutation procedure when we start with a different $R$-charge assignment. Suppose we had a triangle quiver with potential $W=$ $(X Y Z)^{2}$, which requires $R_{X}+R_{Y}+R_{Z}=1$, as in figure 7. As can be inferred from (5.7), 

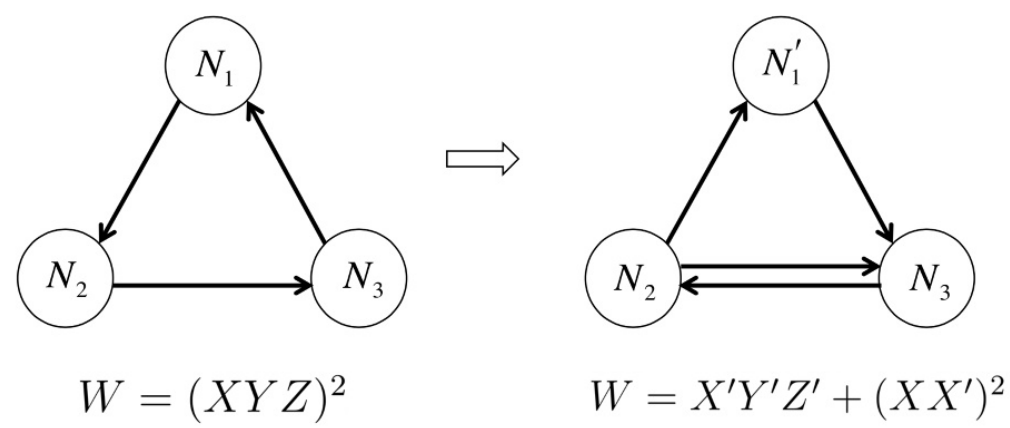

Figure 7. Mutation of a quiver with non-generic superpotential.

the dual quiver has new chiral fields $X^{\prime}, Y^{\prime}, Z^{\prime}$ with $R$-charges $\left(R_{X}^{\prime}, R_{Y}^{\prime}, R_{X}+R_{Y}\right)$ where $R_{X}^{\prime}+R_{Y}^{\prime}=2-R_{X}-R_{Y}$, as well as the original chiral $X$. Because of $R$-charge mismatch, $X$ and $X^{\prime}$ cannot form a mass term $X X^{\prime}$. The superpotential of the mutated theory is generic of type $W=X^{\prime} Y^{\prime} Z^{\prime}+\left(X X^{\prime}\right)^{2}{ }^{9}$

\section{Mutation $\tilde{\mu}_{k}$ on quiver invariant $\left.\Omega_{\mathrm{Q}}\right|_{\mathrm{Inv}}$}

We start with the observation that the alternate mutation rules $\tilde{\mu}^{L, R}$ preserve the quiver invariant regardless of the choices of the chamber or the node, while, as we saw in previous section, $\mu^{L, R}$ preserve Witten index of individual chambers when the node is carefully chosen. Let us start with the explanation of why $\mu_{k}$ cannot preserve the quiver invariants.

Recall that the ordinary mutation rule $\mu$ preserves the total charge $\Gamma=\mu_{k}(\Gamma)$ and, as we demonstrated in previous section, also preserves the index when $k$ and $L, R$ is appropriately chosen. For preservation of the index

$$
\Omega_{\mathrm{Q}}(\zeta)=\Omega_{\mu_{k}^{L, R}(\mathrm{Q})}\left(\mu_{k}^{L, R}(\zeta)\right),
$$

the allowed choice of the mutation node $k$ and the choice between $L$ and $R$ are severely restricted by $\zeta$. The choice becomes clearer when in a given chamber we can take a pair, $\zeta_{k}$ and $\zeta_{k^{\prime}}$, large positive and large negative, respectively and relative to other $\zeta_{l}$ 's: one must perform either $\mu_{k}^{L}$ or $\mu_{k^{\prime}}^{R}$.

Therefore, a chamber of $\mathrm{Q}$ is mapped to a chamber of such a mutated quiver $\mu_{k}(\mathrm{Q})$ but another chamber of $\mathrm{Q}$ is not necessarily mapped to another chamber of the same $\mu_{k}(\mathrm{Q})$. Instead, the latter would be generically mapped to a chamber of a differently mutated quiver $\mu_{l}(\mathrm{Q})$. Generally, the number of chambers for $\mathrm{Q}$ is not necessarily the same as that of $\mu_{k}(\mathrm{Q})$ for a given $k$, so wall-crossing pattern of $\mathrm{Q}$ cannot be the same as that of $\mu_{k}(\mathrm{Q})$ anyhow. It follows that quiver invariant of $Q$ cannot be generally the same as that of $\mu_{k}(\mathrm{Q})$, even though the two share a chamber with the same moduli space topology somewhere in the respective FI constant space.

\footnotetext{
${ }^{9}$ In standard mathematics literature, such possibilities are precluded by assuming absence of 1-cycles and 2-cycles [49, 50].
} 
This happens because the quiver invariant is not a property of the quiver moduli spaces but rather of the quiver itself, or of the gauged quantum mechanics as a whole. On the other hand, the mutation map originates in mathematics literature as a transformation of the quiver diagrams themselves, and it would be strange if there is no definite behavior of the quiver invariants under the mutation maps.

A very strong hint of how quiver invariant should behave under mutation [39] is found in the partition sum expansion of eq. (4.3). The formula implies that quiver invariants of all subquivers should behave like an "elementary" node in the induced quiver $Q /\left\{Q_{p}\right\}$ for any given partition $\mathrm{Q}=\oplus \mathrm{Q}_{p}$. So as far as $\mathrm{Q} /\left\{\mathrm{Q}_{p}\right\}$ goes, the subquiver $\mathrm{Q}_{p}$ of total charge $\Gamma_{p}=\sum_{i} N_{i}^{(p)} \gamma_{i}$ must behave as if it is an elementary node. Therefore, if this expansion makes sense, $\Gamma_{p}$ must be mapped exactly as if $\Gamma_{p}$ belongs to a single node of quiver. This is precisely the mutation maps $\tilde{\mu}$ introduced earlier. Since any quiver can be a subquiver of infinite number of larger quivers, this means that the mutation preserving the quiver invariant has to be the modified one $\tilde{\mu}$

$$
\left.\Omega_{\mathrm{Q}}\right|_{\mathrm{Inv}}=\left.\Omega_{\tilde{\mu}_{k}(\mathrm{Q})}\right|_{\mathrm{Inv}},
$$

which brings us to the assertion at the head of this section.

Recall that the action of $\tilde{\mu}$ is the same as $\mu$ except for the action on the ranks $N_{i}$, as in eq. (2.10). A bonus is that since the quiver invariant is a chamber-independent concept, we need not be careful about $\zeta$ 's, and the restriction on the choice of $k$ does not exist. Therefore, the above holds for any choice of $k$,

$$
\left.\Omega_{\mathrm{Q}}\right|_{\text {Inv }}=\left.\Omega_{\tilde{\mu}_{k}(\mathrm{Q})}\right|_{\text {Inv }}, \quad \text { for all } k
$$

and, for a given quiver with $K$ nodes, one finds as many as $K$ mutated quivers, upon a single mutation step, that shares the same quiver invariant. This is in fact much more powerful and useful statement than the invariance of Witten index under ordinary mutation $\mu$. The latter is mired by the rather complicated choice of the node to be mutated, while the invariance of the quiver invariants under $\tilde{\mu}$ is completely independent of FI constants.

For example, let $\Omega_{a, b, c}^{N, m, n}(\zeta)$ 's be the indices for a cyclic triangle quiver of ranks $N, m, n$ with the opposing intersection numbers $a, b, c$. Using $\tilde{\mu}$ mutation on the first node, we obtain

$$
\left.\Omega_{a, b, c}^{1,1,1}\right|_{\mathrm{Inv}}=\left.\Omega_{a-b c,-b,-c}^{\min [b, c]-1,1,1}\right|_{\mathrm{Inv}} .
$$

For a non-Abelian cyclic triangle, the same procedure gives us

$$
\left.\Omega_{a, b, c}^{N, m, n}\right|_{\operatorname{Inv}}=\left.\Omega_{a-b c,-b,-c}^{\min [b n, c m]-N, m, n}\right|_{\operatorname{Inv}} .
$$

Negative intersection number means flipping of arrows relative to the original quiver, but the overall direction does not matter so we will sometimes flip the intersection numbers altogether. 
Appendix will discuss the validity of this claim for a series of $(N, 1,1)$ quivers which are obtained via $\tilde{\mu}$ mutation from Abelian triangle quivers. Here we simply list the explicit forms of the relevant quiver invariants:

$$
\begin{aligned}
& \left.\Omega_{k^{2}-2, k, k}^{k-1,1,1}\right|_{\operatorname{Inv}}=\left.\Omega_{2, k, k}^{1,1,1}\right|_{\operatorname{Inv}}=k-1, \\
& \left.\Omega_{k^{2}-3, k, k}^{k-1,1,1}\right|_{\text {Inv }}=\left.\Omega_{3, k, 2 k}^{1,1,2}\right|_{\text {Inv }}=\left.\Omega_{3, k, k}^{1,1,1}\right|_{\text {Inv }}=\left[\frac{(k-1)(k-2)}{2}\right] \times(\mathbf{y}+1 / \mathbf{y}), \\
& \left.\Omega_{k(k+1)-3, k, k+1}^{k-1,1,1}\right|_{\text {Inv }}=\left.\Omega_{3,2 k+3, k+1}^{1,2,1}\right|_{\text {Inv }}=\left.\Omega_{3, k, 2 k-1}^{1,1,2}\right|_{\text {Inv }}=\left.\Omega_{3, k, k+1}^{1,1,1}\right|_{\text {Inv }}=\frac{(k-1)(k+2)}{2}, \\
& \left.\Omega_{k^{2}-4, k, k}^{k, 1,1}\right|_{\mathrm{Inv}}=\left.\Omega_{4,3 k, k}^{1,3,1}\right|_{\mathrm{Inv}}=\left.\Omega_{4, k, k}^{1,1,1}\right|_{\mathrm{Inv}} \\
& =\frac{(k-1)(k-2)(k-3)}{6}\left(\mathbf{y}^{2}+1+1 / \mathbf{y}^{2}\right)+\frac{(k-2)\left(k^{2}+1\right)}{2}, \\
& \left.\Omega_{k^{2}+k-4, k, k+1}^{k-1,1,1}\right|_{\text {Inv }}=\left.\Omega_{4,3 k+2, k+1}^{1,3,1}\right|_{\text {Inv }}=\left.\Omega_{4, k, 3 k-1}^{1,1,3}\right|_{\text {Inv }}=\left.\Omega_{4, k, k+1}^{1,1,1}\right|_{\text {Inv }} \\
& =\frac{(k-1)(k-2)(2 k+3)}{6}(\mathbf{y}+1 / \mathbf{y}) \text {, } \\
& \left.\Omega_{k^{2}+2 k-4, k, k+2}^{k-1,1,1}\right|_{\text {Inv }}=\left.\Omega_{4,3 k+8, k+2}^{1,3,1}\right|_{\text {Inv }}=\left.\Omega_{4, k, 3 k-2}^{1,1,3}\right|_{\text {Inv }}=\left.\Omega_{4, k, k+2}^{1,1,1}\right|_{\text {Inv }} \\
& =\frac{(k-1)\left(k^{2}+4 k+6\right)}{6} \text {. }
\end{aligned}
$$

This generalizes to general quivers as follows. Given a quiver, let us concentrate on a mutating node, say of rank $N$, and nodes of rank $m_{i}$ and $n_{p}$, connected to it by, respectively, $c_{i}$ ingoing or $b_{p}$ outgoing arrows. The index may be denoted as

$$
\Omega_{a_{i j} ; a_{i p} ; a_{p q}, b_{p}, c_{i}, \ldots}^{N, m_{i}, n_{p}, \ldots}(\vec{\zeta})
$$

where $b$ 's and $c$ 's denote, respectively, ingoing and outgoing intersection numbers, all taken to be positive, from the mutating node. The three set of numbers encoded in the three matrices, $a_{i j} ; a_{i p} ; a_{p q}$, are intersection numbers among the nodes connected to the mutation node in the initial quiver. With this, the mutation rule for the quiver invariant is

$$
\left.\Omega_{a_{i j} ; a_{i p} ; a_{p q}, b_{p}, c_{i}, \ldots}^{N, m_{i}, \ldots}\right|_{\mathrm{Inv}}=\left.\Omega_{a_{i j} ; a_{i p}-b_{p} c_{i} ; a_{p q},-b_{p},-c_{i}, \ldots}^{\min [b \cdot n, c \cdot m]}\right|_{\mathrm{Inv}} .
$$

As we emphasized already, the quiver invariants are properties of the quivers themselves and therefore we do not need to be selective in choosing mutation nodes. Generally, given a quiver with $K$ number of nodes, there are as many as $K$ mutated quivers whose quiver invariants all agree with the quiver invariant of the original quiver. With such a strong and universal statement, a very tantalizing question that should be explored further is whether this notion of quiver invariants and their invariance under $\tilde{\mu}$ mutations is hidden in the existing cluster algebra structure of quivers, or can be embedded into its generalization. 


\section{Summary}

In this note, we have explored how mutation maps of quiver diagram work to preserve Witten indices and quiver invariants, relying on prototypical examples of triangle quivers.

For Witten indices of $1 \mathrm{~d}$ quiver theories, which are chamber-dependent quantities, mutations are far more restricted than its higher dimensional counterpart. For any given point in $\zeta$ space, only two possible mutations exist, which divides physical chambers further into sub-chambers. The allowed mutation maps a sub-chamber into a physical chamber of the mutated quiver, while disallowed mutation actually fails preserve the Witten index. This identifies a specific chamber of quiver with a specific chamber of the mutated quiver. We have shown how this equality is realized at the level of Witten index expressed as residue integrals for simple class of $(1,1, N)$ triangle quivers.

Quiver invariants, on the other hand, is an intrinsic quantity of the quiver itself rather than its chambers. As such, the complicated (sub-)chamber-dependence should be unnecessary, and we argued that any given node can be mutated to give another quiver of the same quiver invariant. The mutation rule $\tilde{\mu}$ for them differs slightly from those $\mu$ 's used for the Witten indices, in that $\tilde{\mu}$ acts differently on the rank vectors than $\mu$. With a single step of mutation, a quiver with $K$ nodes is mapped to $K \tilde{\mu}$-mutated quivers, therefore. Again we have tested this assertion for the simple classes of triangle quivers, by explicit computations.

\section{Acknowledgments}

We are indebted to Kentaro Hori and Zhao-Long Wang for useful conversations, and Kyungyong Lee for extensive discussions of the Cluster Algebra. H.K. and S-J.L. are grateful to Korea Institute for Advanced Study for hospitality. The work of S.-J.L. is supported in part by NSF grant PHY-1417316. H.K. was supported by the Perimeter Institute for Theoretical Physics. Research at Perimeter Institute is supported by the Government of Canada through Industry Canada and by the Province of Ontario through the Ministry of Economic Development and Innovation. The work of H.K. was made possible through the support of a grant from John Templeton Foundation. The opinions expressed in this publication are those of the author and do not necessarily reflect the views of the John Templeton Foundation.

\section{A Quiver invariants from direct index computations}

We list indices in the four chambers of $(N, 1,1)$ quivers, obtained by $\tilde{\mu}$ mutation from $(1,1,1)$ quivers, computed with the help of HKY routine. This class of quivers comes up to four different chambers in the FI space, and we display index for each chamber. The last item for each quiver is the quiver invariant, extracted by comparing these indices against the Coulombic computations of MPS. ${ }^{10}$

\footnotetext{
${ }^{10}$ The Coulombic computation, which we do not explicitly display here, were obtained using the mathematica codes supplied by Manschot et al. together with ref. [28]. We gratefully acknowledge their generosity for making the code public.
} 
In the MPS expansion, quiver invariants of all subquivers are left as unknown input parameters, so that the comparison against HKY computation fixes these quantity. For the MPS expansion, for which a mathematica package supplied in ref. [28] is used, the quantity being computed is usually Poincare polynomial rather than the index. However, the expansion formula itself should be applicable to the index as well, because purely Coulombic states are neutral under $\mathrm{U}(1)_{R}$ while the states counted by quiver invariants are neutral under $\mathrm{SU}(2)_{R}$. The actual vacua are obtained by simple tensor product of these two classes of states, and their quantum numbers are already manifest individually.

The following confirms the quiver invariants of all $(2,1,1)$ quivers that appear in eq. (6.5), up to $k=9$, and eq. (6.6), up to $k=8$, by direct computations. To extract quiver invariant of given $(N, 1,1)$ quiver, by comparing HKY index against MPS's partition sum, one ends up computing quiver invariants of $(n, 1,1)$ quivers for all $n \leq N$ recursively. However, for all of examples below, $(1,1,1)$ quivers happen to carry no nontrivial quiver invariant. For Abelian cyclic quivers, the geometric characterization of the quiver invariant in refs. $[25,26]$ is applicable, so that the quiver invariant is null whenever there is a chamber of null Higgs moduli space. For this reason, we chose not to display the indices of the Abelian version.

- $(2,1,1)$-Quiver with intersection numbers $(2 k, k, 3)$

$$
\begin{aligned}
& \Omega_{6,3,3}^{2,1,1}=\left(\begin{array}{l}
0 \\
0 \\
0 \\
0
\end{array}\right. \\
& \left.\Omega_{6,3,3}^{2,1,1}\right|_{\text {Inv }}=1 / \mathbf{y}+\mathbf{y} \\
& \Omega_{8,4,3}^{2,1,1}=\left(\begin{array}{l}
-1 / \mathbf{y}^{5}-2 / \mathbf{y}^{3}-1 / \mathbf{y}-\mathbf{y}-2 \mathbf{y}^{3}-\mathbf{y}^{5} \\
2 / \mathbf{y}+2 \mathbf{y} \\
2 / \mathbf{y}+2 \mathbf{y} \\
2 / \mathbf{y}+2 \mathbf{y}
\end{array}\right. \\
& \left.\Omega_{8,4,3}^{2,1,1}\right|_{\text {Inv }}=3 / \mathbf{y}+3 \mathbf{y} \\
& \Omega_{10,5,3}^{2,1,1}=\left(\begin{array}{l}
-1 / \mathbf{y}^{9}-2 / \mathbf{y}^{7}-4 / \mathbf{y}^{5}-6 / \mathbf{y}^{3}-2 / \mathbf{y}-2 \mathbf{y}-6 \mathbf{y}^{3}-4 \mathbf{y}^{5}-2 \mathbf{y}^{7}-\mathbf{y}^{9} \\
5 / \mathbf{y}+5 \mathbf{y} \\
5 / \mathbf{y}+5 \mathbf{y} \\
5 / \mathbf{y}+5 \mathbf{y}
\end{array}\right. \\
& \left.\Omega_{10,5,3}^{2,1,1}\right|_{\text {Inv }}=6 / \mathbf{y}+6 \mathbf{y} \\
& \Omega_{12,6,3}^{2,1,1}=\left(\begin{array}{l}
-1 / \mathbf{y}^{13}-2 / \mathbf{y}^{11}-4 / \mathbf{y}^{9}-6 / \mathbf{y}^{7}-9 / \mathbf{y}^{5}-11 / \mathbf{y}^{3} \\
\quad-3 / \mathbf{y}-3 \mathbf{y}-11 \mathbf{y}^{3}-9 \mathbf{y}^{5}-6 \mathbf{y}^{7}-4 \mathbf{y}^{9}-2 \mathbf{y}^{11}-\mathbf{y}^{13} \\
9 / \mathbf{y}+9 \mathbf{y} \\
9 / \mathbf{y}+9 \mathbf{y} \\
9 / \mathbf{y}+9 \mathbf{y}
\end{array}\right.
\end{aligned}
$$




$$
\begin{aligned}
& \left.\Omega_{12,6,3}^{2,1,1}\right|_{\text {Inv }}=10 / \mathbf{y}+10 \mathbf{y} \\
& \Omega_{14,7,3}^{2,1,1}=\left(\begin{array}{c}
-1 / \mathbf{y}^{17}-2 / \mathbf{y}^{15}-4 / \mathbf{y}^{13}-6 / \mathbf{y}^{11}-9 / \mathbf{y}^{9}-12 / \mathbf{y}^{7} \\
-15 / \mathbf{y}^{5}-17 / \mathbf{y}^{3}-4 / \mathbf{y}-4 \mathbf{y}-17 \mathbf{y}^{3}-15 \mathbf{y}^{5} \\
-12 \mathbf{y}^{7}-9 \mathbf{y}^{9}-6 \mathbf{y}^{11}-4 \mathbf{y}^{13}-2 \mathbf{y}^{15}-\mathbf{y}^{17} \\
14 / \mathbf{y}+14 \mathbf{y} \\
14 / \mathbf{y}+14 \mathbf{y} \\
14 / \mathbf{y}+14 \mathbf{y}
\end{array}\right. \\
& \left.\Omega_{14,7,3}^{2,1,1}\right|_{\text {Inv }}=15 / \mathbf{y}+15 \mathbf{y} \\
& \Omega_{16,8,3}^{2,1,1}=\left(\begin{array}{l}
-1 / \mathbf{y}^{21}-2 / \mathbf{y}^{19}-4 / \mathbf{y}^{17}-6 / \mathbf{y}^{15}-9 / \mathbf{y}^{13}-12 / \mathbf{y}^{11}-16 / \mathbf{y}^{9} \\
-19 / \mathbf{y}^{7}-22 / \mathbf{y}^{5}-24 / \mathbf{y}^{3}-5 / \mathbf{y}-5 \mathbf{y}-24 \mathbf{y}^{3}-22 \mathbf{y}^{5}-19 \mathbf{y}^{7} \\
-16 \mathbf{y}^{9}-12 \mathbf{y}^{11}-9 \mathbf{y}^{13}-6 \mathbf{y}^{15}-4 \mathbf{y}^{17}-2 \mathbf{y}^{19}-\mathbf{y}^{21} \\
20 / \mathbf{y}+20 \mathbf{y} \\
20 / \mathbf{y}+20 \mathbf{y} \\
20 / \mathbf{y}+20 \mathbf{y}
\end{array}\right. \\
& \left.\Omega_{16,8,3}^{2,1,1}\right|_{\text {Inv }}=21 / \mathbf{y}+21 \mathbf{y} \\
& \Omega_{18,9,3}^{2,1,1}=\left(\begin{array}{c}
-1 / \mathbf{y}^{25}-2 / \mathbf{y}^{23}-4 / \mathbf{y}^{21}-6 / \mathbf{y}^{19}-9 / \mathbf{y}^{17}-12 / \mathbf{y}^{15} \\
-16 / \mathbf{y}^{13}-20 / \mathbf{y}^{11}-24 / \mathbf{y}^{9}-27 / \mathbf{y}^{7}-30 / \mathbf{y}^{5}-32 / \mathbf{y}^{3}-6 / \mathbf{y} \\
-6 \mathbf{y}-32 \mathbf{y}^{3}-30 \mathbf{y}^{5}-27 \mathbf{y}^{7}-24 \mathbf{y}^{9}-20 \mathbf{y}^{11}-16 \mathbf{y}^{13} \\
\quad-12 \mathbf{y}^{15}-9 \mathbf{y}^{17}-6 \mathbf{y}^{19}-4 \mathbf{y}^{21}-2 \mathbf{y}^{23}-\mathbf{y}^{25} \\
27 / \mathbf{y}+27 \mathbf{y} \quad \\
27 / \mathbf{y}+27 \mathbf{y} \\
27 / \mathbf{y}+27 \mathbf{y}
\end{array}\right. \\
& \left.\Omega_{18,9,3}^{2,1,1}\right|_{\text {Inv }}=28 / \mathbf{y}+28 \mathbf{y}
\end{aligned}
$$

- $(2,1,1)$-Quiver with intersection numbers $(2 k+3, k+1,3)$

$$
\begin{aligned}
\Omega_{9,4,3}^{2,1,1} & =\left(\begin{array}{l}
1 / \mathbf{y}^{6}+2 / \mathbf{y}^{4}+4 / \mathbf{y}^{2}+10+4 \mathbf{y}^{2}+2 \mathbf{y}^{4}+\mathbf{y}^{6} \\
1 / \mathbf{y}^{2}+7+\mathbf{y}^{2} \\
6 \\
6
\end{array}\right. \\
\left.\Omega_{9,4,3}^{2,1,1}\right|_{\text {Inv }} & =5 \\
\Omega_{11,5,3}^{2,1,1} & =\left(\begin{array}{l}
1 / \mathbf{y}^{10}+2 / \mathbf{y}^{8}+4 / \mathbf{y}^{6}+6 / \mathbf{y}^{4}+8 / \mathbf{y}^{2}+18+8 \mathbf{y}^{2}+6 \mathbf{y}^{4}+4 \mathbf{y}^{6}+2 \mathbf{y}^{8}+\mathbf{y}^{10} \\
10 \\
10
\end{array}\right. \\
\left.\Omega_{11,5,3}^{2,1,1}\right|_{\operatorname{Inv}} & =9
\end{aligned}
$$




$$
\begin{aligned}
& \Omega_{13,6,3}^{2,1,1}=\left(\begin{array}{l}
1 / \mathbf{y}^{14}+2 / \mathbf{y}^{12}+4 / \mathbf{y}^{10}+6 / \mathbf{y}^{8}+9 / \mathbf{y}^{6}+11 / \mathbf{y}^{4}+13 / \mathbf{y}^{2} \\
+28+13 \mathbf{y}^{2}+11 \mathbf{y}^{4}+9 \mathbf{y}^{6}+6 \mathbf{y}^{8}+4 \mathbf{y}^{10}+2 \mathbf{y}^{12}+\mathbf{y}^{14} \\
1 / \mathbf{y}^{2}+16+\mathbf{y}^{2} \\
15 \\
15
\end{array}\right. \\
& \left.\Omega_{13,6,3}^{2,1,1}\right|_{\text {Inv }}=14 \\
& \Omega_{15,7,3}^{2,1,1}=\left(\begin{array}{c}
\begin{array}{c}
1 / \mathbf{y}^{18}+2 / \mathbf{y}^{16}+4 / \mathbf{y}^{14}+6 / \mathbf{y}^{12}+9 / \mathbf{y}^{10}+12 / \mathbf{y}^{8} \\
+15 / \mathbf{y}^{6}+17 / \mathbf{y}^{4}+19 / \mathbf{y}^{2}+40+19 \mathbf{y}^{2}+17 \mathbf{y}^{4}+15 \mathbf{y}^{6} \\
\quad+12 \mathbf{y}^{8}+9 \mathbf{y}^{10}+6 \mathbf{y}^{12}+4 \mathbf{y}^{14}+2 \mathbf{y}^{16}+\mathbf{y}^{18}
\end{array} \\
\begin{array}{c}
1 / \mathbf{y}^{2}+22+\mathbf{y}^{2} \\
21
\end{array} \\
21
\end{array}\right. \\
& \left.\Omega_{15,7,3}^{2,1,1}\right|_{\text {Inv }}=20
\end{aligned}
$$

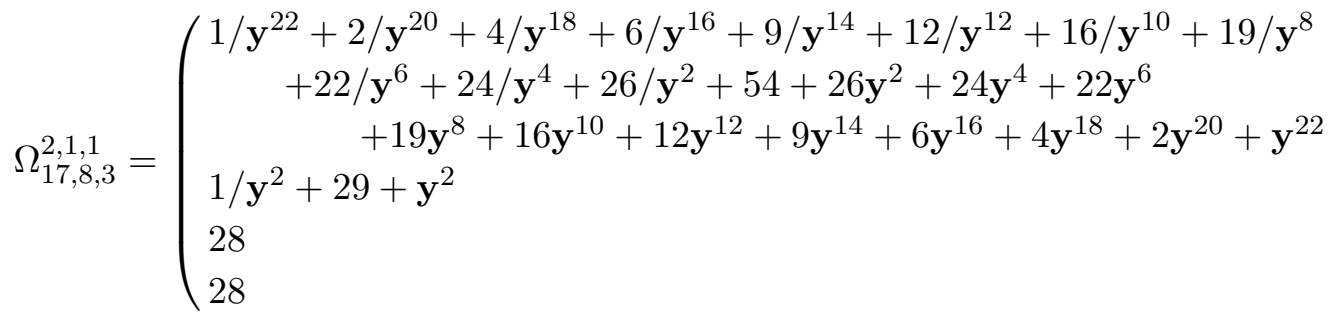

$$
\begin{aligned}
& \left.\Omega_{17,8,3}^{2,1,1}\right|_{\text {Inv }}=27
\end{aligned}
$$

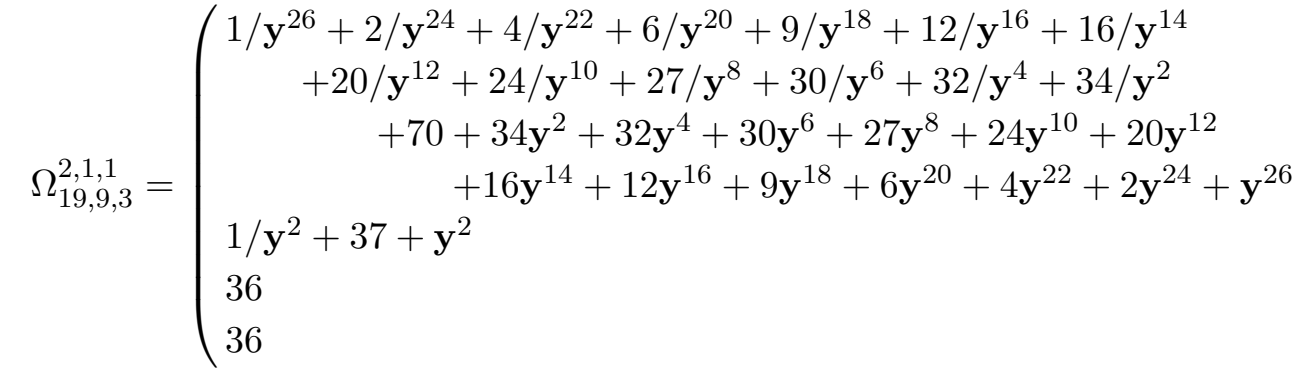

$$
\begin{aligned}
& \left.\Omega_{19,9,3}^{2,1,1}\right|_{\text {Inv }}=35
\end{aligned}
$$

- $(2,1,1)$-Quiver with intersection numbers $(2 k-1, k, 3)$

$$
\begin{aligned}
\Omega_{5,3,3}^{2,1,1} & =\left(\begin{array}{l}
6 \\
6 \\
1 / \mathbf{y}^{2}+7+\mathbf{y}^{2} \\
1 / \mathbf{y}^{2}+7+\mathbf{y}^{2}
\end{array}\right. \\
\left.\Omega_{5,3,3}^{2,1,1}\right|_{\text {Inv }} & =5
\end{aligned}
$$




$$
\begin{aligned}
& \Omega_{7,4,3}^{2,1,1}=\left(\begin{array}{l}
1 / \mathbf{y}^{4}+2 / \mathbf{y}^{2}+13+2 \mathbf{y}^{2}+\mathbf{y}^{4} \\
10 \\
1 / \mathbf{y}^{2}+11+\mathbf{y}^{2} \\
1 / \mathbf{y}^{2}+11+\mathbf{y}^{2}
\end{array}\right. \\
& \left.\Omega_{7,4,3}^{2,1,1}\right|_{\text {Inv }}=9 \\
& \Omega_{9,5,3}^{2,1,1}=\left(\begin{array}{l}
1 / \mathbf{y}^{8}+2 / \mathbf{y}^{6}+4 / \mathbf{y}^{4}+6 / \mathbf{y}^{2}+22+6 \mathbf{y}^{2}+4 \mathbf{y}^{4}+2 \mathbf{y}^{6}+\mathbf{y}^{8} \\
15 \\
1 / \mathbf{y}^{2}+16+\mathbf{y}^{2} \\
1 / \mathbf{y}^{2}+16+\mathbf{y}^{2}
\end{array}\right. \\
& \left.\Omega_{9,5,3}^{2,1,1}\right|_{\operatorname{Inv}}=14 \\
& \Omega_{11,6,3}^{2,1,1}=\left(\begin{array}{l}
1 / \mathbf{y}^{12}+2 / \mathbf{y}^{10}+4 / \mathbf{y}^{8}+6 / \mathbf{y}^{6}+9 / \mathbf{y}^{4}+11 / \mathbf{y}^{2} \\
\quad+33+11 \mathbf{y}^{2}+9 \mathbf{y}^{4}+6 \mathbf{y}^{6}+4 \mathbf{y}^{8}+2 \mathbf{y}^{10}+\mathbf{y}^{12} \\
21 \\
1 / \mathbf{y}^{2}+22+\mathbf{y}^{2} \\
1 / \mathbf{y}^{2}+22+\mathbf{y}^{2}
\end{array}\right. \\
& \left.\Omega_{11,6,3}^{2,1,1}\right|_{\text {Inv }}=20
\end{aligned}
$$

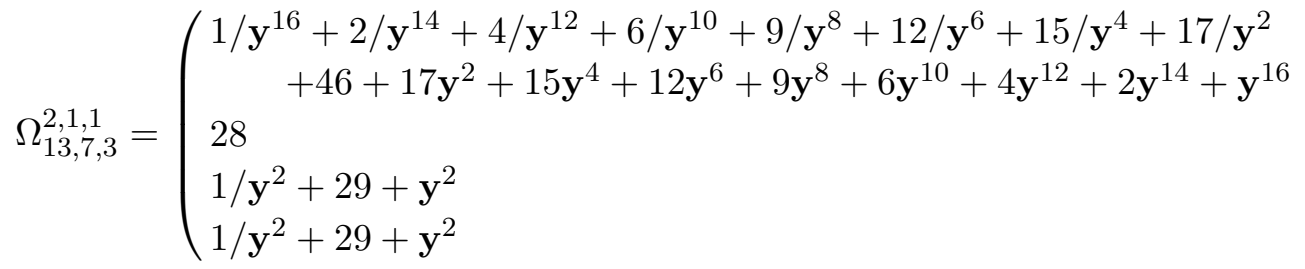

$$
\begin{aligned}
& \left.\Omega_{13,7,3}^{2,1,1}\right|_{\text {Inv }}=27
\end{aligned}
$$

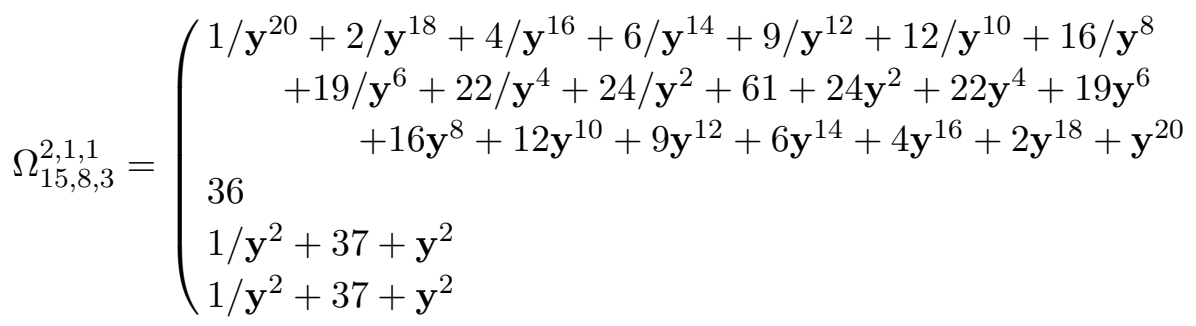

$$
\begin{aligned}
& \left.\Omega_{15,8,3}^{2,1,1}\right|_{\text {Inv }}=35
\end{aligned}
$$

In all examples above, the computed quiver invariants agree with predictions from the mutations $\tilde{\mu}$, displayed in section 6 .

We have also confirmed the quiver invariants in all $(3,1,1)$ quivers that appear in eqs. (6.7)-(6.9) up to $k=9$, via direct computations along the same line as the $(2,1,1)$ cases above. 
Open Access. This article is distributed under the terms of the Creative Commons Attribution License (CC-BY 4.0), which permits any use, distribution and reproduction in any medium, provided the original author(s) and source are credited.

\section{References}

[1] N. Seiberg and E. Witten, Electric-magnetic duality, monopole condensation and confinement in $N=2$ supersymmetric Yang-Mills theory, Nucl. Phys. B 426 (1994) 19 [Erratum ibid. B 430 (1994) 485] [hep-th/9407087] [INSPIRE].

[2] N. Seiberg and E. Witten, Monopoles, duality and chiral symmetry breaking in $N=2$ supersymmetric QCD, Nucl. Phys. B 431 (1994) 484 [hep-th/9408099] [INSPIRE].

[3] F. Ferrari and A. Bilal, The strong coupling spectrum of the Seiberg-Witten theory, Nucl. Phys. B 469 (1996) 387 [hep-th/9602082] [INSPIRE].

[4] K.-M. Lee and P. Yi, Dyons in $N=4$ supersymmetric theories and three pronged strings, Phys. Rev. D 58 (1998) 066005 [hep-th/9804174] [INSPIRE].

[5] D. Bak, C.-k. Lee, K.-M. Lee and P. Yi, Low-energy dynamics for $1 / 4$ BPS dyons, Phys. Rev. D 61 (2000) 025001 [hep-th/9906119] [INSPIRE].

[6] J.P. Gauntlett, N. Kim, J. Park and P. Yi, Monopole dynamics and BPS dyons $N=2$ super Yang-Mills theories, Phys. Rev. D 61 (2000) 125012 [hep-th/9912082] [INSPIRE].

[7] M. Stern and P. Yi, Counting Yang-Mills dyons with index theorems, Phys. Rev. D 62 (2000) 125006 [hep-th/0005275] [INSPIRE].

[8] F. Denef, Supergravity flows and D-brane stability, JHEP 08 (2000) 050 [hep-th/0005049] [INSPIRE].

[9] F. Denef, Quantum quivers and Hall/hole halos, JHEP 10 (2002) 023 [hep-th/0206072] [INSPIRE].

[10] F. Denef and G.W. Moore, Split states, entropy enigmas, holes and halos, JHEP 11 (2011) 129 [hep-th/0702146] [INSPIRE].

[11] M. Kontsevich and Y. Soibelman, Stability structures, motivic Donaldson-Thomas invariants and cluster transformations, arXiv:0811.2435 [INSPIRE].

[12] D. Gaiotto, G.W. Moore and A. Neitzke, Four-dimensional wall-crossing via three-dimensional field theory, Commun. Math. Phys. 299 (2010) 163 [arXiv:0807.4723] [INSPIRE].

[13] D. Gaiotto, G.W. Moore and A. Neitzke, Wall-crossing, Hitchin Systems and the WKB Approximation, arXiv:0907.3987 [INSPIRE].

[14] D. Gaiotto, G.W. Moore and A. Neitzke, Framed BPS States, Adv. Theor. Math. Phys. 17 (2013) 241 [arXiv: 1006.0146] [INSPIRE].

[15] J. de Boer, S. El-Showk, I. Messamah and D. Van den Bleeken, Quantizing $N=2$ Multicenter Solutions, JHEP 05 (2009) 002 [arXiv: 0807.4556] [INSPIRE].

[16] J. Manschot, B. Pioline and A. Sen, Wall Crossing from Boltzmann Black Hole Halos, JHEP 07 (2011) 059 [arXiv: 1011.1258] [INSPIRE].

[17] J. Manschot, B. Pioline and A. Sen, A fixed point formula for the index of multi-centered $N=2$ black holes, JHEP 05 (2011) 057 [arXiv:1103.1887] [INSPIRE]. 
[18] H. Kim, J. Park, Z. Wang and P. Yi, Ab Initio Wall-Crossing, JHEP 09 (2011) 079 [arXiv: 1107.0723] [INSPIRE].

[19] A. Sen, Equivalence of three wall-crossing formulae, Commun. Num. Theor. Phys. 6 (2012) 601 [arXiv:1112.2515] [INSPIRE].

[20] S. Kachru and J. McGreevy, Supersymmetric three cycles and supersymmetry breaking, Phys. Rev. D 61 (2000) 026001 [hep-th/9908135] [INSPIRE].

[21] S. Lee and P. Yi, Framed BPS States, Moduli Dynamics and Wall-Crossing, JHEP 04 (2011) 098 [arXiv: 1102.1729] [INSPIRE].

[22] M. Alim, S. Cecotti, C. Cordova, S. Espahbodi, A. Rastogi and C. Vafa, BPS Quivers and Spectra of Complete $N=2$ Quantum Field Theories, Commun. Math. Phys. 323 (2013) 1185 [arXiv: 1109.4941] [INSPIRE].

[23] M. Alim, S. Cecotti, C. Cordova, S. Espahbodi, A. Rastogi and C. Vafa, $\mathcal{N}=2$ quantum field theories and their BPS quivers, Adv. Theor. Math. Phys. 18 (2014) 27 [arXiv:1112.3984] [INSPIRE].

[24] I. Bena, M. Berkooz, J. de Boer, S. El-Showk and D. Van den Bleeken, Scaling BPS Solutions and pure-Higgs States, JHEP 11 (2012) 171 [arXiv:1205.5023] [INSPIRE].

[25] S.-J. Lee, Z.-L. Wang and P. Yi, Quiver Invariants from Intrinsic Higgs States, JHEP 07 (2012) 169 [arXiv:1205.6511] [INSPIRE].

[26] S.-J. Lee, Z.-L. Wang and P. Yi, BPS States, Refined Indices and Quiver Invariants, JHEP 10 (2012) 094 [arXiv: 1207.0821] [INSPIRE].

[27] M. Reineke, The Harder-Narasimhan system in quantum groups and cohomology of quiver moduli, Invent. Math. 152 (2003) 349 [math/0204059].

[28] J. Manschot, B. Pioline and A. Sen, On the Coulomb and Higgs branch formulae for multi-centered black holes and quiver invariants, JHEP 05 (2013) 166 [arXiv:1302.5498] [INSPIRE].

[29] J. Manschot, B. Pioline and A. Sen, The Coulomb Branch Formula for Quiver Moduli Spaces, arXiv:1404.7154 [INSPIRE].

[30] K. Hori, H. Kim and P. Yi, Witten Index and Wall Crossing, JHEP 01 (2015) 124 [arXiv: 1407.2567] [INSPIRE].

[31] S.-J. Lee, Z.-L. Wang and P. Yi, Abelianization of BPS Quivers and the Refined Higgs Index, JHEP 02 (2014) 047 [arXiv: 1310.1265] [INSPIRE].

[32] C. Cordova and S.-H. Shao, Counting Trees in Supersymmetric Quantum Mechanics, arXiv:1502.08050 [INSPIRE].

[33] H. Kim, Scaling Behaviour of Quiver Quantum Mechanics, arXiv:1503.02623 [INSPIRE].

[34] C. Cordova and S.-H. Shao, Asymptotics of Ground State Degeneracies in Quiver Quantum Mechanics, arXiv: 1503.03178 [INSPIRE].

[35] F. Benini, D.S. Park and P. Zhao, Cluster algebras from dualities of $2 d N=(2,2)$ quiver gauge theories, arXiv:1406.2699 [INSPIRE].

[36] F. Benini, C. Closset and S. Cremonesi, Comments on 3d Seiberg-like dualities, JHEP 10 (2011) 075 [arXiv:1108.5373] [INSPIRE]. 
[37] C. Closset, Seiberg duality for Chern-Simons quivers and D-brane mutations, JHEP 03 (2012) 056 [arXiv:1201.2432] [INSPIRE].

[38] D. Xie, Three dimensional Seiberg-like duality and tropical cluster algebra, arXiv:1311.0889 [INSPIRE].

[39] J. Manschot, B. Pioline and A. Sen, Generalized quiver mutations and single-centered indices, JHEP 01 (2014) 050 [arXiv: 1309.7053] [INSPIRE].

[40] C. Cordova and S.-H. Shao, An Index Formula for Supersymmetric Quantum Mechanics, arXiv: 1406.7853 [INSPIRE].

[41] C. Hwang, J. Kim, S. Kim and J. Park, General instanton counting and 5d SCFT, arXiv: 1406.6793 [INSPIRE].

[42] A. Sen, Arithmetic of Quantum Entropy Function, JHEP 08 (2009) 068 [arXiv:0903.1477] [INSPIRE].

[43] A. Sen, How Do Black Holes Predict the Sign of the Fourier Coefficients of Siegel Modular Forms?, Gen. Rel. Grav. 43 (2011) 2171 [arXiv: 1008.4209] [InSPIRE].

[44] N. Seiberg, Electric-magnetic duality in supersymmetric nonAbelian gauge theories, Nucl. Phys. B 435 (1995) 129 [hep-th/9411149] [INSPIRE].

[45] L.C. Jeffrey and F.C. Kirwan, Localization for nonabelian group actions, Topology 34 (1995) 291 [alg-geom/9307001].

[46] M. Brion and M. Vergne, Arrangement og hyperplanes I: Rational functions and Jeffrey-Kirwan residue, Ann. Sci. ENS 32 (1999) 715 [math/9903178].

[47] A. Szenes and M. Vergne, Toric reduction and a conjecture of batyrev and materov, Invent. Math. 158 (2004) 453 [math/0306311].

[48] F. Benini, R. Eager, K. Hori and Y. Tachikawa, Elliptic Genera of $2 d \mathcal{N}=2$ Gauge Theories, Commun. Math. Phys. 333 (2015) 1241 [arXiv:1308.4896] [INSPIRE].

[49] H. Derksen, J. Weyman and A. Zelevinsky, Quivers with potentials and their representations. I: Mutations, Sel. Math 14 (2008) 59 [arXiv:0704.0649].

[50] B. Keller and D Yang, Derived equivalences from mutations of quivers with potential, Adv. Math. 226 (2011) 2118 [arXiv:0906.0761]. 